\title{
Second-order accurate finite volume schemes with the discrete maximum principle for solving Richards' equation on unstructured meshes
}

\author{
D. Svyatskiya,1, K. Lipnikov ${ }^{\mathrm{a}}$ \\ ${ }^{a}$ Applied Mathematics and Plasma Physics Group, Theoretical Division, \\ Los Alamos National Laboratory, Los Alamos, NM 87545, USA
}

\begin{abstract}
Richards's equation describes steady-state or transient flow in a variably saturated medium. For a medium having multiple layers of soils that are not aligned with coordinate axes, a mesh fitted to these layers is no longer orthogonal and the classical two-point flux approximation finite volume scheme is no longer accurate. We propose new secondorder accurate nonlinear finite volume (NFV) schemes for the head and pressure formulations of Richards' equation. We prove that the discrete maximum principles hold for both formulations at steady-state which mimics similar properties of the continuum solution. The second-order accuracy is achieved using high-order upwind algorithms for the relative permeability. Numerical simulations of water infiltration into a dry soil show significant advantage of the second-order NFV schemes over the first-order NFV schemes even on coarse meshes. Since explicit calculation of the Jacobian matrix becomes prohibitively expensive for high-order schemes due to build-in reconstruction and slope limiting algorithms, we study numerically the preconditioning strategy introduced recently in [21] that uses a stable approximation of the continuum Jacobian. Numerical simulations show that the new preconditioner reduces computational cost up to 2-3 times in comparison with the conventional preconditioners.
\end{abstract}

Keywords: Richards' equation, discrete maximum principle, nonlinear finite volume scheme, unstructured meshes

\section{Introduction}

In a wide range of water resource management and hydrology applications modeling of subsurface flows in variably saturated porous media is the important part of overall simulations. In many cases these flows are modeled assuming that the movement of the gas phase can be neglected. This assumption leads to the flow model described by Richards' equation [31] which has less unknowns in comparison with a two-phase formulation. The flexibility and appeal of this model is evident in more complex systems, such as non-isothermal three-phase (ice, water, air) systems modeling hydrology in degrading permafrost $[16,35]$. However, this simplification also makes Richards' equation a strongly nonlinear elliptic-parabolic partial differential equation (PDE), creating significant challenges for accurate discretization schemes and efficient linear and nonlinear solvers.

Early work on numerical methods for Richards' equation focused on orthogonal Cartesian grids with isotropic or grid-aligned anisotropic absolute permeabilities, for which the two-point flux approximation (TPFA) of the Darcy flux is accurate. It was shown that the implicit discretization in time of the mixed formulation of Richards' equation, in concert with the TPFA in space, conserves mass, see e.g. [8]. The upwinding of the relative permeability based

Email addresses: dasvyat@lanl.gov (D. Svyatskiy), lipnikov@lanl.gov (K. Lipnikov) 
on the flow direction is essential to capture its strong nonlinearity, particularly with infiltration into a dry soil, see [5]. In this setting other important properties of the continuum model are naturally preserved in a numerical solution such as the symmetry and discrete maximum principles for the hydraulic head and aqueous pressure. Moreover, the discrete nonlinear operator has structure typical for a symmetric M-matrix which is beneficial for linear and nonlinear solvers; although, the Jacobian matrix is a non-symmetric matrix. The main drawback of the TPFA approach is that it is limited to the case of K-orthogonal meshes in which normals to mesh faces (edges in 2D) are aligned with the principle directions of the permeability tensor. In complex simulations, the computational mesh has to be fitted to surface topography, subsurface geology, and graded towards small engineering and natural subsurface structures. These requirements make impossible to build accurate discrete models using the TPFA framework.

The multi-point flux approximation (MPFA) method improves accuracy on general meshes by using more than two points in the flux approximation stencil [1]. For diffusion problems, the MPFA method leads to second-order accurate schemes on general meshes that are only conditionally stable [17] and conditionally monotone [30]. Monotone schemes guarantee some solution bounds, such as positivity of conventration, but still allow the numerical solution to oscillate. Several methods are closely related to the MPFA method, such as the control-volume distributed (CVD) method [13] and the variant of the mixed finite element method presented in [39]. The recent CVD scheme with the full pressure support increases significantly the stability region [14]; still, in practice, lack of unconditional maximum principles may lead to nonphysical oscillations in both the head and pressure, hence, to incorrect sign of the numerical Darcy flux and wrong prediction of a contaminant transport. Other advanced discretization methods, such as the mimetic finite difference [20], hybrid finite volume, mixed finite volume [11], and mixed finite element [6] methods are unconditionally stable on unstructured shape-regular meshes. Unfortunately, these methods lead to schemes with the discrete maximum principles only when certain relations between the mesh and permeability tensors hold.

A new finite volume scheme that has accuracy of the MPFA family of schemes but preserves important properties of the continuum solution (like the TPFA schemes) on arbitrarily polytopal meshes was proposed in [24] for linear diffusion equations. This scheme uses a specially designed solution-dependent stencil for the flux approximation; hence, it is nonlinear even for linear equations. For this reason, we refer to it as the nonlinear finite volume (NFV) scheme. Since Richards' equation is already nonlinear, the additional nonlinearity due to the discretization may be acceptable for simulations contingent to thorough numerical investigation. Here, we extend this NFV scheme to Richards' equation and study numerically and theoretically its properties. Note that many NFV schemes for linear diffusion equations have been proposed during the last decade staring with the seminal papers by Bertolazzi and Manzini [26] for a scalar tensor and Le Potier [18] for a full tensor. The NFV schemes have been applied successfully for numerical solution of multiphase flows [28, 33] and well driven flows [10] where they were coupled with the Peaceman model. It is important to distinguish between positivity preserving NFV schemes, e.g. [18], and NFV schemes with the discrete maximum principles (DMPs), e.g. [24]. Only in the latter case the maximum and minimum principles are satisfied simultaneously which guarantees an oscillation-free numerical solution.

Many existing NFV schemes introduce auxiliary unknowns at mesh vertices or on mesh edges whose values are interpolated linearly or nonlinearly from solution values in the neighboring cells, see $[9,40,29]$ and references therein. Although, the auxiliary unknowns are not included into the final system of discrete equations, the interpolation has to be performed on each nonlinear iteration. To reduce cost of an accurate interpolation on an unstructured mesh, a few interpolation-free NFV schemes were proposed and analyzed in [22, 23] for diffusion and advection-diffusion equations with smooth coefficients. However, for problems with discontinuous permeability tensors, a smaller number of auxiliary unknowns on interface edges (faces in 3D) is still needed for these schemes but with quite simple interpolation formulas. For instance, the NFV scheme in [24] uses a compact linearity-preserving two-point interpolation formula based on the harmonic averaging point introduced in [2]. Note that convergence analysis of NFV schemes preserving the DMP is often based on a coercivity assumption, like in [12].

In this paper, we consider the head and pressure formulations of Richards' equation. We extend the NFV scheme from [24] to both formulations and also merge it with the second-order upwind algorithm for the relative permeability. 
To the best of our knowledge, the resulting schemes are the first ones for this model which are the second-order accurate in the global $L_{2}$ norm on general polygonal meshes and preserve the DMP either for the hydraulic head or aqueous pressure. Near solution extrema accuracy of the schemes reduces to the first order due to the slope-limiting procedure. Numerical simulations of water infiltration into a dry soil show significant advantage of the second-order NFV schemes over the first-order NFV schemes and the TPFA schemes.

The second-order upwind algorithm is based on a linear reconstruction of pressure inside each mesh cell. For numerical stability and in order to have the DMP, the reconstructed solution is limited, typically in vicinity of a wetting front. We demonstrate robust performance of the tensorial limiter [23] that avoids over-limitation of the reconstructed gradient and hence provides better accuracy compared to a simpler approach such as the Barth-Jesperson limiter [4]. Even on coarse meshes, the new NFV schemes are more accurate than the first-order NFV schemes on twice refined meshes. This justifies the overhead caused by the reconstruction and limiting algorithms.

In general, slope limiters are not differentiable and cause problems for Newton and Newton-type solvers based on the Jacobian matrix. In addition, nonlinearity of the discretization makes significant contribution to the cost of calculating this matrix. In this paper, we test the different preconditioning strategy proposed recently in [21]. This strategy builds a preconditioner using the continuum Jacobian and stable discretization schemes for all its terms. Our numerical experiments indicate consistent positive impact of the new preconditioner on the convergence rate of the Anderson acceleration method when compared to the standard approach, when the matrix of the discrete system with frozen nonlinear coefficients is used as a preconditioner. In half of the experiments, the computational time was reduced roughly twice.

The paper outline is as follows. In Section 2, we introduce Richards' equation and summarize mathematical properties of its solution. In Section 3, we describe two NFV schemes for the head and pressure formulations of Richards' equation and prove a few mathematical properties for discrete solutions. In Section 4, the describe briefly the Anderson acceleration nonlinear method. The second-order convergence rate towards the analytic 1D solution is demonstrated in Section 5. In Section 6, we discuss numerical results obtained with the proposed schemes for a few challenging problems of water infiltration into a layered dry soil described by a piecewise constant anisotropic absolute permeability tensor.

\section{Richards equation}

Let $\Omega$ be a two-dimensional polygonal domain with the Lipschitz boundary $\Gamma$. The mixed form of Richards' equation for the unknown hydraulic head $h[\mathrm{~m}]$ and Darcy velocity $\mathbf{q}\left[\mathrm{m} \cdot \mathrm{s}^{-1}\right]$ reads:

$$
\frac{\partial\left(\rho \phi s\left(p_{c}\right)\right)}{\partial t}+\operatorname{div}(\rho \mathbf{q})=0, \quad \mathbf{q}=-\mathbf{K}(x, z) k\left(s\left(p_{c}\right)\right) \frac{\rho g}{\mu} \nabla h .
$$

Here $g$ is the magnitude of gravity vector $\mathrm{g}=(0,-g)^{T}, s\left(p_{c}\right)[-]$ is the water saturation function of capillary pressure $p_{c}=p_{a t m}-p, p[\mathrm{~Pa}]$ is the aqueous pressure, and $h=p /(\rho g)+z$. The first equation is the mass balance equation, the second one is the nonlinear Darcy law. The medium properties are described by porosity $\phi[-]$, absolute permeability tensor $\mathbf{K}\left[\mathrm{m}^{2}\right]$, and relative permeability function $k(s)[-], 0<k(s) \leqslant 1$. The fluid properties are described by density $\rho\left[\mathrm{kg} \cdot \mathrm{m}^{-3}\right]$ and viscosity $\mu[\mathrm{Pa} \cdot \mathrm{s}]$.

In this study, we consider constant $\rho$ and $\mu$. We assume that $\Omega$ consists of a few different soils $\Omega_{s}$ characterized by full symmetric positive definite tensors $\mathbf{K}_{s}(x, z)$ and different porosities $\phi_{s}$. We assume that $\phi_{s}$ is constant inside each soil. A water retention model establishes the relationship between pressure and saturation functions, $s\left(p_{c}\right)$, and provides a closed form analytic expression for the relative permeability as a function of pressure, $k(p)=k(s(p))$. Typically, the relative permeability is a non-decreasing function of $p$ :

$$
\frac{\partial k}{\partial p} \geqslant 0
$$


In this paper we consider the relative permeability model defined by the van Genuchten and Mualem equations, see [37]:

$$
\left.k(s)=\sqrt{s}\left[1-\left[1-s^{1 / m}\right]\right]^{m}\right]^{2}, \quad s\left(p_{c}\right)=\left[1+\left(\alpha\left|p_{c}\right|\right)^{n}\right]^{-m},
$$

where $\alpha, n$ and $m=\frac{n-1}{n}$ are parameters of the model.

Richards' equation can be also written in terms of the pressure function $p$ using relationship $h=p /(\rho g)+z$ where the $z$-axis points in the direction opposite to the gravity direction $\mathrm{g}$ :

$$
\frac{\partial(\rho \phi s)}{\partial t}+\operatorname{div}(\rho \mathbf{q})=0, \quad \mathbf{q}=-\mathbf{K} \frac{k(p)}{\mu}(\nabla p-\rho \mathbf{g}) .
$$

Both equations have a few remarkable mathematical properties. The steady-state solution to (1) satisfies the strong form of the maximum principle, e.g. the hydraulic head has no internal extrema. The steady state-solution to (4) satisfies the strong form of the maximum principle inside each soil provided that $\mathbf{K}_{s}$ is constant [27]. Since, the maximum principle holds typically in a vicinity of the steady-state solution, a numerical scheme that respects it will lead to a more accurate simulation, especially when solution dynamics is smooth.

The fluid flow is driven by gravity and boundary conditions. We split the boundary $\Gamma$ into two parts, $\Gamma_{D}$ and $\Gamma_{N}$, and set

$$
\begin{aligned}
p & =p_{D}(\mathbf{x}) \quad \text { on } \Gamma_{D} \\
\mathbf{q} \cdot \mathbf{n} & =q_{N}(\mathbf{x}) \quad \text { on } \Gamma_{N} .
\end{aligned}
$$

The system (4)-(5) is closed by initial conditions for the pressure $p$. In many practical simulations the initial pressure profile is unknown; therefore, it has to be computed in a preliminary stage which is referred to as the steady-state calculation.

\section{A nonlinear finite volume scheme}

Two second-order accurate NFV schemes described below preserve the maximum principles on a general polygonal mesh. This is due to a special approximation of the Darcy fluxes on mesh edges. The first scheme (Sections 3.2-3.3) uses head unknowns, while the second one (Sections 3.4-3.5) uses pressure unknowns.

\subsection{Mesh notation}

Let $\mathcal{T}$ be a conformal mesh composed of $\mathcal{N}_{c}$ polygonal cells $c$ with edges $e$. We assume that $\mathcal{T}$ is edge-connected, i.e. it cannot be split into two sub-meshes having no common edges. We also assume that the interfaces between different soils are approximated by mesh edges so that each mesh cell contains only one soil.

Let $|c|$ denote the area of cell $c$ and $|e|$ be the length of edge $e$. We denote by $\mathbf{n}_{c}\left(\right.$ also, $\left.\mathbf{n}_{c, e}\right)$ the exterior normal vector to $\partial c$ and by $\mathbf{n}_{e}$ the normal vector to edge $e$ fixed once and for all, such that $\left|\mathbf{n}_{e}\right|=\left|\mathbf{n}_{c, e}\right|=|e|$. On a boundary edge, the vector $\mathbf{n}_{e}$ is always exterior. The set of boundary edges is denoted by $\mathcal{E}_{\Gamma}$, and its proper subset of edges belonging to $\Gamma_{N}$ is denoted by $\mathcal{E}_{\Gamma}^{N}$. Let $\mathbf{x}_{c}$ denote centroid of cell $c$, and $\mathcal{E}(c)$ denote the set of edges of $c$. Finally, let $\mathbf{x}_{e}$ be the center of edge $e$. For simplicity, we assume that the absolute permeability tensor is constant inside each cell; otherwise, we approximate it by a constant tensor $\mathbf{K}_{c}$. For a cell $c_{i}$, we shall write $\mathbf{K}_{i}$ instead of $\mathbf{K}_{c_{i}}$ and $\mathbf{x}_{i}$ instead of $\mathbf{x}_{c_{i}}$. 


\subsection{Scheme I: Approximation of the Darcy flux inside homogeneous soil}

The first NFV scheme uses one degree of freedom, $h_{c}$, per cell $c$ collocated at point $\mathbf{x}_{c}$. For a cell $c_{i}$, we shall write $h_{i}$ instead of $h_{c_{i}}$. Integrating the mass balance equation in (1) over $c$ and using the divergence theorem, we obtain the following exact equations:

$$
\rho \phi_{c} \frac{\partial}{\partial t} \int_{c} s \mathrm{~d} V+\rho \sum_{e \in \partial c} \xi_{c, e} \mathbf{q}_{e} \cdot \mathbf{n}_{e}=0, \quad \mathbf{q}_{e}=\frac{1}{|e|} \int_{e} \mathbf{q} \mathrm{d} S
$$

where $\xi_{c, e}=\left(\mathbf{n}_{e} \cdot \mathbf{n}_{c, e}\right) /|e|^{2}$ and $\mathbf{q}_{e} \cdot \mathbf{n}_{e}$ is the unique Darcy flux on edge $e$. The Neumann boundary conditions define Darcy fluxes on boundary edges $e \in \mathcal{E}_{\Gamma}^{N}$ that can be inserted immediately in equation (6). The Dirichlet boundary condition on edge $e \in \mathcal{E}_{\Gamma}^{D}$ is imposed using the following average head:

$$
h_{e}=\frac{1}{|e|} \int_{e}\left(\frac{p_{D}}{\rho g}+z\right) \mathrm{d} S .
$$

A finite volume scheme is obtained by replacing continuum fluxes $\mathbf{q}_{e} \cdot \mathbf{n}_{e}$ by their discrete approximations. We derive a new formula for the discrete Darcy flux that uses head values in a few neighboring cells, mostly the closes neighbors of $c$. The practical implementation of this scheme leads to an algebraic problem those sparsity structure corresponds to a compact stencil, e.g. the five-point stencil on a logically rectangular mesh.

For en edge $e$ shared by two cells $c_{i}$ and $c_{j}$, we introduce vector $\mathbf{t}_{i j}=\mathbf{x}_{c_{j}}-\mathbf{x}_{c_{i}}$ that connects their centroids. We assume that for every edge $e$ of cell $c_{i}$ and the associated co-normal vector $\ell_{e, i}=\mathbf{K}_{i} \mathbf{n}_{c_{i}, e}$ there exist two vectors $\mathbf{t}_{i j}$ and $\mathbf{t}_{i k}$ (see the left picture in Fig. 1) such that

$$
\ell_{e, i}=\alpha_{i j} \mathbf{t}_{i j}+\alpha_{i k} \mathbf{t}_{i k}, \quad \alpha_{i j} \geqslant 0, \quad \alpha_{i k} \geqslant 0 .
$$

This positive decomposition implies that the angles between pairs of vectors $\mathbf{t}_{i j}, \mathbf{t}_{i k}$, then $\mathbf{t}_{i j}, \ell_{e, i}$, and finally $\mathbf{t}_{i k}, \ell_{e, i}$ are smaller than $180^{\circ}$ which is true for almost all meshes used in practice. In a few rare exceptional cases, we can consider additional neighbors of cells $c_{i}$ to enrich the set of vectors $\mathbf{t}_{i k}$.

Let us consider edge $e$ shared by cells $c_{i}, c_{j} \in \Omega_{s}$, i.e. the permeability tensor changes smoothly across edge $e$. The case of a discontinuous permeability tensor is considered in the next subsection. We assume that $\mathbf{n}_{e}$ points from $c_{i}$ to $c_{j}$. When cell $c_{i}$ is adjacent to the domain boundary $\Gamma_{D}$, one should replace $c_{j}$ and $\mathbf{x}_{j}$ in the formulas below with the boundary edge $e$ and its barycenter $\mathbf{x}_{e}$. Let us break the normal component of the Darcy flux into two factors:

$$
\mathbf{q}_{e} \cdot \mathbf{n}_{c_{i}, e}=\left(-\frac{\rho}{\mu} k\right)\left(\widetilde{\mathbf{q}}_{e} \cdot \mathbf{n}_{c_{i}, e}\right), \quad \widetilde{\mathbf{q}}_{e} \cdot \mathbf{n}_{c_{i}, e}=\mathbf{K}_{i} \nabla h \cdot \mathbf{n}_{c_{i}, e}=\nabla h \cdot\left(\mathbf{K}_{i} \mathbf{n}_{c_{i}, e}\right)=\nabla h \cdot \ell_{e, i} .
$$

Let $k_{e}$ denote some approximation of the relative permeability on edge $e$ discussed later. Inside $\Omega_{s}$, accurate approximation of the co-normal derivative of $h$ can be performed with finite differences:

$$
\begin{aligned}
\widetilde{\mathbf{q}}_{e} \cdot \mathbf{n}_{c_{i}, e} \equiv \nabla h \cdot \ell_{e, i} & =\alpha_{i j} \nabla h \cdot \mathbf{t}_{i j}+\alpha_{i k} \nabla h \cdot \mathbf{t}_{i k} \\
& =\alpha_{i j}\left(h_{j}-h_{i}\right)+\alpha_{i k}\left(h_{k}-h_{i}\right)+O\left(|e|^{2}\right) .
\end{aligned}
$$

The first auxiliary numerical flux denoted by $\widetilde{q}_{e}^{h, 1}$ is obtained by dropping out the approximation term $O\left(|e|^{2}\right)$. The formula for the numerical Darcy mass flux reads:

$$
q_{e}^{h, 1}=-\frac{\rho}{\mu} k_{e} \widetilde{q}_{e}^{h, 1}=-\frac{\rho}{\mu} k_{e}\left[\alpha_{i j}\left(h_{j}-h_{i}\right)+\alpha_{i k}\left(h_{k}-h_{i}\right)\right] .
$$



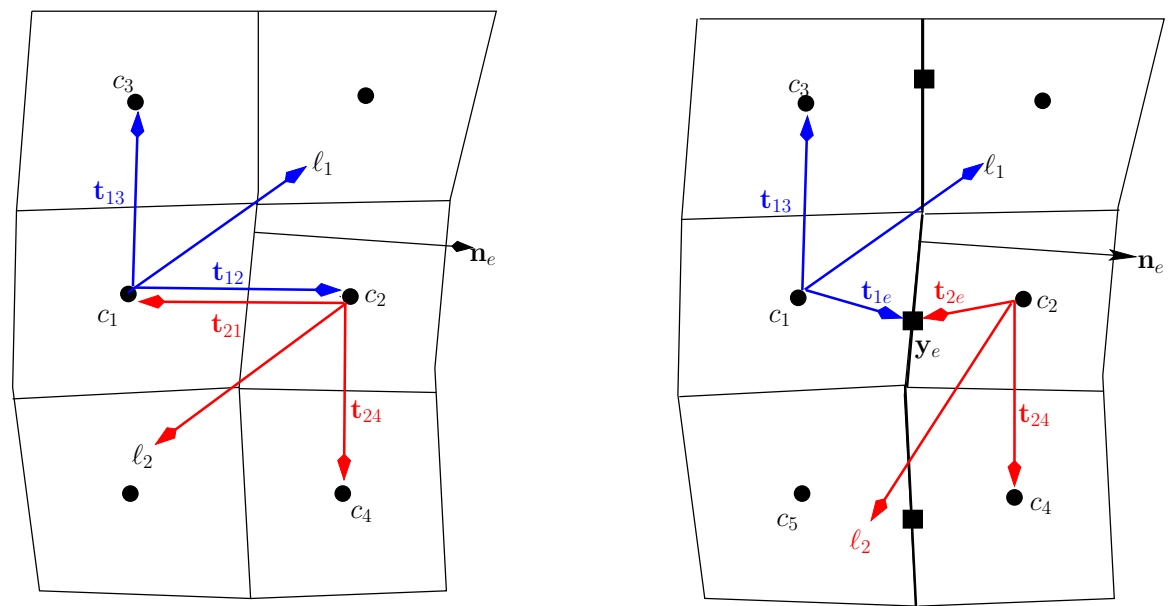

Figure 1: Co-normals $\ell_{1}$ and $\ell_{2}$ and related pairs $\mathbf{t}_{i j}, \mathbf{t}_{i k}$ for homogeneous medium (left) and heterogeneous medium (right). The bold edges indicate the material interface. The bold circles indicate location of collocation points. The bold squares indicate location of harmonic averaging points.

We will refer to $\widetilde{q}_{e}^{h, 1}$ as the one-sided auxiliary flux from cell $c_{i}$ to cell $c_{j}$. Similarly, we can derive the one-sided auxiliary flux from $c_{j}$ to $c_{i}$ denoted by $\widetilde{q}_{e}^{h, 2}$. A unique flux is defined as a special combination of these one-sided fluxes which we explain using the left panel in Fig. 1. The first one-sided auxiliary flux from cell $c_{1}$ to cell $c_{2}$ is obtained by setting $i=1, j=2$, and $k=3$ in formula (9):

$$
\widetilde{q}_{e}^{h, 1}=\alpha_{12}\left(h_{2}-h_{1}\right)+\alpha_{13}\left(h_{3}-h_{1}\right) .
$$

Similarly, the second one-sided auxiliary flux, now from cell $c_{2}$ to cell $c_{1}$, is obtained by setting $i=2, j=1$, and $k=4$ in formula (9):

$$
\widetilde{q}_{e}^{h, 2}=\alpha_{21}\left(h_{1}-h_{2}\right)+\alpha_{24}\left(h_{4}-h_{2}\right) .
$$

The unique auxiliary numerical flux on edge $e$ is a linear combination of the one-sided fluxes:

$$
\begin{aligned}
\widetilde{q}_{e}^{h}=\mu_{1} \widetilde{q}_{e}^{h, 1}+\mu_{2}\left(-\widetilde{q}_{e}^{h, 2}\right) & =\mu_{1}\left(\alpha_{12}\left(h_{2}-h_{1}\right)+\alpha_{13}\left(h_{3}-h_{1}\right)\right) \\
& -\mu_{2}\left(\alpha_{21}\left(h_{1}-h_{2}\right)+\alpha_{24}\left(h_{4}-h_{2}\right)\right) .
\end{aligned}
$$

The selection of non-negative weights $\mu_{1}$ and $\mu_{2}$ is one of the key-points of the proposed NFV scheme. They are defined by the system of two equations. The first equation comes from the approximation viewpoint and states that the linear combination (10) must be convex:

$$
\mu_{1}+\mu_{2}=1 \text {. }
$$

To find the second equation, we follow $[24,34]$ and require that the contributions from both side in (10) are equal:

$$
\mu_{1}\left|\widetilde{q}_{e}^{h, 1}\right|=\mu_{2}\left|\widetilde{q}_{e}^{h, 2}\right| .
$$

To solve system (11)-(12), we have to consider two cases. If $\left|\widetilde{q}_{e}^{h, 1}\right|+\left|\widetilde{q}_{e}^{h, 2}\right|=0$, then both contributions are already balanced; hence, we can take $\mu_{1}=\mu_{2}=0.5$. Otherwise, the unique solution is given by

$$
\mu_{1}=\frac{\left|\widetilde{q}_{e}^{h, 2}\right|}{\left|\widetilde{q}_{e}^{h, 1}\right|+\left|\widetilde{q}_{e}^{h, 2}\right|}, \quad \mu_{2}=\frac{\left|\widetilde{q}_{e}^{h, 1}\right|}{\left|\widetilde{q}_{e}^{h, 1}\right|+\left|\widetilde{q}_{e}^{h, 2}\right|},
$$


If $\widetilde{q}_{e}^{h, 1} \widetilde{q}_{e}^{h, 2} \leqslant 0$, we can show that

$$
\widetilde{q}_{e}^{h}=\frac{2 \widetilde{q}_{e}^{h, 1}\left|\widetilde{q}_{e}^{h, 2}\right|}{\left|\widetilde{q}_{e}^{h, 1}\right|+\left|\widetilde{q}_{e}^{h, 2}\right|}=-\frac{2\left|\widetilde{q}_{e}^{h, 1}\right| \widetilde{q}_{e}^{h, 2}}{\left|\widetilde{q}_{e}^{h, 1}\right|+\left|\widetilde{q}_{e}^{h, 2}\right|} ;
$$

hence, there exists two algebraically equivalent representations of the unique flux:

$$
\widetilde{q}_{e}^{h}=2 \mu_{1}\left(\alpha_{12}\left(h_{2}-h_{1}\right)+\alpha_{13}\left(h_{3}-h_{1}\right)\right)=A_{12}\left(h_{2}-h_{1}\right)+A_{13}\left(h_{3}-h_{1}\right)
$$

and

$$
\widetilde{q}_{e}^{h}=-2 \mu_{2}\left(\alpha_{21}\left(h_{1}-h_{2}\right)+\alpha_{24}\left(h_{4}-h_{2}\right)\right)=A_{21}\left(h_{2}-h_{1}\right)+A_{24}\left(h_{2}-h_{4}\right),
$$

where $A_{12}=2 \mu_{1} \alpha_{12}, A_{13}=2 \mu_{1} \alpha_{13}, A_{21}=2 \mu_{2} \alpha_{21}$ and $A_{24}=2 \mu_{2} \alpha_{24}$.

If $\widetilde{q}_{e}^{h, 1} \widetilde{q}_{e}^{h, 2}>0$, the resulting unique flux is zero, which may lead to the algebraic problem with a reducible matrix. In order to avoid this potential degeneracy, we re-group terms in (10) to separate the head difference between cells $c_{1}$ and $c_{2}$ :

$$
\widetilde{q}_{e}^{h}=\mu_{1} \bar{q}_{e}^{h, 1}+\mu_{2}\left(-\bar{q}_{e}^{h, 2}\right)+\left(\mu_{1} \alpha_{12}+\mu_{2} \alpha_{21}\right)\left(h_{2}-h_{1}\right),
$$

where $\bar{q}_{e}^{h, 1}=\alpha_{13}\left(h_{3}-h_{1}\right)$ and $\bar{q}_{e}^{h, 2}=\alpha_{24}\left(h_{4}-h_{2}\right)$. New weights $\mu_{1}$ and $\mu_{2}$ are selected to balance these fluxes:

$$
\left|\bar{q}_{e}^{h, 1}\right| \mu_{1}=\left|\bar{q}_{e}^{h, 2}\right| \mu_{2}
$$

The unique solution to (11), (17) is given by

$$
\mu_{1}=\frac{\left|\bar{q}_{e}^{h, 2}\right|}{\left|\bar{q}_{e}^{h, 1}\right|+\left|\bar{q}_{e}^{h, 2}\right|}, \quad \mu_{2}=\frac{\left|\bar{q}_{e}^{h, 1}\right|}{\left|\bar{q}_{e}^{h, 1}\right|+\left|\bar{q}_{e}^{h, 2}\right|} .
$$

Inserting this into formula (16), we obtain

$$
\widetilde{q}_{e}^{h}=\frac{\bar{q}_{e}^{h, 1}\left|\bar{q}_{e}^{h, 2}\right|-\left|\bar{q}_{e}^{h, 1}\right| \bar{q}_{e}^{h, 2}}{\left|\bar{q}_{e}^{h, 1}\right|+\left|\bar{q}_{e}^{h, 2}\right|}+\left(\mu_{1} \alpha_{12}+\mu_{2} \alpha_{21}\right)\left(h_{1}-h_{2}\right) .
$$

Let $A_{12}=\mu_{1} \alpha_{12}+\mu_{2} \alpha_{21}$. If $\bar{q}_{e}^{h, 1} \bar{q}_{e}^{h, 2}>0$, we obtain the two-point flux formula:

$$
\widetilde{q}_{e}^{h}=\left(\mu_{1} \alpha_{12}+\mu_{2} \alpha_{21}\right)\left(h_{1}-h_{2}\right)=A_{12}\left(h_{2}-h_{1}\right),
$$

which implies the non-zero contribution to the matrix and guarantees its irreducibility. If $\bar{q}_{e}^{h, 1} \bar{q}_{e}^{h, 2} \leqslant 0$, we have again two equivalent algebraic representations of the unique auxiliary flux:

$$
\widetilde{q}_{e}^{h}=2 \mu_{1} \bar{q}_{e}^{h, 1}+A_{12}\left(h_{2}-h_{1}\right)=A_{13}\left(h_{3}-h_{1}\right)+A_{12}\left(h_{2}-h_{1}\right)
$$

and

$$
\widetilde{q}_{e}^{h}=-2 \mu_{2} \bar{q}_{e}^{h, 2}+A_{12}\left(h_{2}-h_{1}\right)=A_{24}\left(h_{2}-h_{4}\right)+A_{12}\left(h_{2}-h_{1}\right) .
$$

The corresponding Darcy mass fluxes are obtained by multiplying the above expressions by $\left(-\rho k_{e} / \mu\right)$.

Remark 3.1. If $\Omega_{h}$ is a Voronoi mesh and $\mathbf{K}_{s}$ is a scalar tensor, then the directions $\mathbf{t}_{i j}$ are co-linear to vectors $\mathbf{n}_{e}$. Hence, some coefficients $\alpha_{i j}$ equals to 0 . As the result of this, only the third term in the right-hand side of equation (16) is non-zero. Then, $\widetilde{q}_{e}^{h}$ is the conventional two-point flux.

The coefficients $A_{12}, A_{13}, A_{21}$, and $A_{24}$ are non-negative by construction. They depend on fluxes and hence on solution values at neighboring cells. Due to two representations for the unique flux, we can use formula (14) or (19) in the mass balance equation for cell $c_{1}$ and formula (15) or (20) in the mass balance equation for cell $c_{2}$. These fluxes contributes non-negative entries to the main diagonal of the stiffness matrix. The off-diagonal entries of this matrix are non-positive and the row sum of matrix entries is non-negative. Using the Dirichlet boundary conditions and our assumption of the mesh edge-connectivity, we can easily show that the resulting matrix is an M-matrix. 


\subsection{Scheme I: Approximation of the Darcy flux on interface between soils}

In this section we consider the case when cells $c_{1}$ and $c_{2}$ share edge $e$ that is on the interface between different soils. In other words, we consider the case of a discontinuous absolute permeability tensor. We cannot approximate accurately a directional derivative across such an edge with finite differences. To resolve this problem, we introduce a harmonic average point on every edge where the absolute permeability tensor has a sharp jump. Let us denote the line containing $e$ by $L_{e}$ and consider a continuous piecewise linear function $\mathcal{R}(\mathbf{x})$ such that

$$
\mathcal{R}\left(\mathbf{x}_{c_{1}}\right)=h_{c_{1}}, \quad \mathcal{R}\left(\mathbf{x}_{c_{2}}\right)=h_{c_{2}},
$$

and the normal component of the related flux is continuous:

$$
\mathbf{K}_{c_{1}} \nabla \mathcal{R}(\mathbf{x}) \cdot \mathbf{n}_{e}=\mathbf{K}_{c_{2}} \nabla \mathcal{R}(\mathbf{x}) \cdot \mathbf{n}_{e} .
$$

Then, there exist the unique harmonic average point $\mathbf{y}_{e} \in L_{e}$ such that the head value, $h_{e}$, at this point can be computed accurately as follows [2]:

$$
h_{e}:=\mathcal{R}\left(\mathbf{y}_{e}\right)=\alpha_{e} h_{c_{1}}+\left(1-\alpha_{e}\right) h_{c_{2}},
$$

where

$$
\alpha_{e}=\frac{d_{2, e} \mathbf{n}_{e} \cdot\left(\mathbf{K}_{c_{1}} \mathbf{n}_{e}\right)}{d_{2, e} \mathbf{n}_{e} \cdot\left(\mathbf{K}_{c_{1}} \mathbf{n}_{e}\right)+d_{1, e} \mathbf{n}_{e} \cdot\left(\mathbf{K}_{c_{2}} \mathbf{n}_{e}\right)}, \quad 0 \leqslant \alpha_{e} \leqslant 1,
$$

and $d_{i, e}$ is the distance from point $\mathbf{x}_{c_{i}}$ to line $L_{e}$. Note that $\alpha_{e}$ depends only on the permeability tensors and mesh geometry.

Now the directional derivatives of $h$ can be approximated using head values at the harmonic averaging points. The right panel in Fig. 1 illustrates the case when the harmonic averaging point $\mathbf{y}_{e}$ is inside edge $e$ so that the vectors $\mathbf{t}_{1 e}$ and $\mathbf{t}_{2 e}$ do not cross the interface between different soils and the respective directional derivatives are accurate. The formula for the resulting final flux $q_{e}^{h}$ involves both $h_{c_{i}}$ and $h_{e}$. The latter can be eliminated using convex combination (21) without increasing the stencil size and preserving the M-matrix property of the resulting algebraic system.

Remark 3.2. In a few rare cases, the harmonic averaging point $\mathbf{y}_{e}$ is located outside mesh edge e. This does not break the formal order of convergence of the NFV scheme but indicates that the piecewise linear approximation $\mathcal{R}(\mathbf{x})$ is possibly used outside of its validity region which may increase local approximation error.

\subsection{Scheme II: Approximation of the diffusion part of the Darcy flux}

For the pressure formulation (4), we split the Darcy flux into the diffusion and gravity parts:

$$
\mathbf{q}_{e} \cdot \mathbf{n}_{e}=-\left(\frac{\rho}{\mu} k\right)\left(\widetilde{\mathbf{q}}_{e}^{d} \cdot \mathbf{n}_{e}-\widetilde{\mathbf{q}}_{e}^{g} \cdot \mathbf{n}_{e}\right), \quad \widetilde{\mathbf{q}}_{e}^{d} \cdot \mathbf{n}_{e}=\mathbf{K}_{i} \nabla p \cdot \mathbf{n}_{e} \quad \widetilde{\mathbf{q}}_{e}^{g} \cdot \mathbf{n}_{e}=\rho \mathbf{K}_{i} \mathbf{g} \cdot \mathbf{n}_{e} .
$$

The diffusion part of the flux is approximated using formulas in Sections 3.2-3.3 and replacing the hydraulic head by the aqueous pressure. The approximation of the gravity part is described in the next subsection.

\subsection{Scheme II: Approximation of the gravity part of the Darcy flux}

In the most general case, we have two different approximations of the gravity part of the Darcy flux:

$$
\widetilde{q}_{e, 1}^{g}=\rho \mathbf{K}_{1} \mathbf{g} \cdot \mathbf{n}_{c_{1}, e} \quad \text { and } \quad \widetilde{q}_{e, 2}^{g}=\rho \mathbf{K}_{2} \mathbf{g} \cdot \mathbf{n}_{c_{2}, e} .
$$


To enforce the mass conservation and continuity of the normal component of the Darcy flux, a unique value for this flux is required. To derive this value, we apply the idea described in the previous section. Two weights $\mu_{1}^{g}$ and $\mu_{2}^{g}$ are selected to balance the fluxes from both sides and the unique flux is defined as follows:

$$
\widetilde{q}_{e}^{g}=\mu_{1}^{g} \widetilde{q}_{e, 1}^{g}+\mu_{2}^{g}\left(-\widetilde{q}_{e, 2}^{g}\right) .
$$

If $\left|\widetilde{q}_{e, 1}^{g}\right|+\left|\widetilde{q}_{e, 2}^{g}\right|=0$, then $\mu_{1}^{g}=\mu_{2}^{g}=0.5$. Otherwise, we balance the contributions from both sides in a similar way as in the case of the diffusion fluxes. The most common case is when $\widetilde{q}_{e, 1}^{g} \widetilde{q}_{e, 2}^{g}<0$ which means that the "gravity fluxes" have the same signs with respect to the fixed normal $\mathbf{n}_{e}$. Strong anisotropy of the permeability tensor may results in $\widetilde{q}_{e, 1}^{g} \widetilde{q}_{e, 2}^{g} \geqslant 0$, i.e. zero unique flux. From an algebraic viewpoint, zero flux is harmless, since it contributes only to the right hand side. Therefore, in both cases, we define the weights $\mu_{i}$ as follows:

$$
\mu_{1}^{g}=\frac{\left|\widetilde{q}_{e, 2}^{g}\right|}{\left|\widetilde{q}_{e, 1}^{g}\right|+\left|\widetilde{q}_{e, 2}^{g}\right|}, \quad \mu_{2}=1-\mu_{1}^{g}=\frac{\left|\widetilde{q}_{e, 1}^{g}\right|}{\left|\widetilde{q}_{e, 1}^{g}\right|+\left|\widetilde{q}_{e, 2}^{g}\right|} .
$$

If $\mathbf{K}_{1}$ and $\mathbf{K}_{2}$ are scalar tensors, then the resulting gravity flux is the gravity flux with the harmonically averaged permeability tensor.

\subsection{Both schemes: Approximation of the relative permeability}

To complete the definition of the discrete Darcy flux, the relative permeability $k_{e}$ has to be defined for every edge $e$. In many existing approaches, $k_{e}$ is set to the value of the relative permeability in the upwind cell for edge $e$. The upwind direction is given by the sign of flux $q_{e}^{h}$. If cell $c_{u, e}$ is the upwind cell for edge $e$, then $k_{e}=k\left(p_{c_{u, e}}\right)$. This approach treats effectively the relative permeability as a piece-wise constant mesh function and leads to an accurate dynamics of a wetting front propagating into a dry soil. We verified with numerical experiments that this approximation results in a scheme with the first-order convergence rate on a sequence of refined meshes. Hereafter, we refer to such a scheme as the first-order NFV scheme or simply as the NFV I scheme.

To increase accuracy, we propose to employ a linear reconstruction of the relative permeability inside cells which should lead to more accurate approximations on mesh edges. There are three physical quantities which can be used as a basis for this reconstruction: the cell-centered relative permeability, pressure, or hydraulic head. Among those, the relative permeability exhibits the strongest nonlinear behavior which may impact convergence of a nonlinear solver and accuracy of the approximation. Therefore, the reconstruction algorithm based on the cell-centered pressures or heads looks more promising. In this work, we selected pressure for the reconstruction algorithm along with the slopelimiting algorithm proposed in [23]. The linear reconstruction $\mathcal{R}_{c}(\mathbf{x})$ in cell $c$ is defined as follows:

$$
\mathcal{R}_{c}(\mathbf{x})=p_{c}+\mathbf{G}_{c} \cdot\left(\mathbf{x}-\mathbf{x}_{c}\right) \quad \forall \mathbf{x} \in c,
$$

where $\mathbf{G}_{c}$ is a reconstructed limited gradient. Hereafter, we refer to such a scheme as the second-order NFV scheme or simply as the NFV II scheme.

Since $p_{c}$ is collocated at the barycenter of cell $c$, this reconstruction preserves the mean value of the pressure for any choice of $\mathbf{G}_{c}$. The gradient is derived based on approximation arguments and limited using stability arguments. To avoid its over-limitation and increase accuracy of the reconstruction, we use the simplified version of the tensorial limiter [23]. For cell $c$, let $\Sigma_{c}$ be the set of neighboring cells and boundary edges on $\Gamma$. An admissible gradient $\mathbf{G}_{c}$ should satisfy the following inequalities:

$$
\min _{c^{\prime \prime} \in \Sigma_{c}} p_{c^{\prime}} \leqslant p_{c}+\mathbf{G}_{c} \cdot\left(\mathbf{x}_{c^{\prime}}-\mathbf{x}_{c}\right) \leqslant \max _{c^{\prime \prime} \in \Sigma_{c}} p_{c^{\prime \prime}} \quad \forall c^{\prime}, c^{\prime \prime} \in \Sigma_{c} .
$$

Since a different equation is considered in [23], the additional constraints proposed there are not required for Richards' equation. 
Let $\mathcal{G}_{c}$ be the set of all admissible gradients satisfying (23). Then, the limited gradient, still denoted by $\mathbf{G}_{c}$, is computed by solving the following constraint minimization problem:

$$
\mathbf{G}_{c}=\arg \min _{\tilde{\mathbf{G}}_{c} \in \mathcal{G}_{c}} \mathcal{J}_{c}\left(\tilde{\mathbf{G}}_{c}\right),
$$

where the functional $\mathcal{J}_{c}$ penalizes deviation from the neighboring pressure values,

$$
\mathcal{J}_{c}\left(\tilde{\mathbf{G}}_{c}\right)=\frac{1}{2} \sum_{c^{\prime} \in \Sigma_{c}}\left[p_{c}+\tilde{\mathbf{G}}_{c} \cdot\left(\mathbf{x}_{c^{\prime}}-\mathbf{x}_{c}\right)-p_{c^{\prime}}\right]^{2} .
$$

This minimization problem has a unique solution provided that set $\Sigma_{c}$ is sufficiently large, see [23] for more details. The limited gradient guarantees that the reconstructed pressure values on edges of cell $c$ are bounded by maximum and minimum of the selected neighboring pressure values. After $\mathbf{G}_{c}$ is computed, the relative permeability $k_{e}$ is defined as follows:

$$
k_{e}=k\left(s\left(\mathcal{R}_{c_{u, e}}\left(\mathbf{x}_{\mathbf{e}}\right)\right)\right)
$$

where $c_{u, e}$ is the upwind cell. In the next subsection we demonstrate importance of the limiting procedure for the discrete maximum principle.

\subsection{Local maximum principle}

Here, we study properties of the proposed NFV schemes. But first, let us revisit properties of the continuum solution. According to [15], the continuum maximum principle has not been extended to nonlinear parabolic equations; however, there are a few results for steady-state solutions. For a steady-state infiltration problem, the hydraulic head $h$ achieves its maximum on the boundary of $\Omega$. If, in addition, the absolute permeability is constant in subdomains $\Omega_{s} \subset \Omega$, we may also have the maximum principle for pressure $p$ inside each $\Omega_{s}$ but not globally in $\Omega$ [27]. Let us show that our schemes preserve these properties for steady-state solutions.

Theorem 3.1 (head formulation). Let $\left\{h_{c}\right\}_{c \in \Omega_{h}}$ be the steady-state solution of the NFV scheme for the head formulation of the infiltration problem, i.e. $q_{N} \leqslant 0$. Furthermore, let the mesh be edge-connected. Then, the discrete head solution does not have internal extrema.

Proof. The stiffness matrix of our scheme has weak diagonal dominance in rows. Since the mesh is edge-connected and $A_{12}>0$ for each mesh edge, this matrix is also irreduciable. Hence, the discrete maximum and minimum principles for the solution follow immediately from the algebraic result in $[7,36]$.

Theorem 3.2 (pressure formulation). Let $\left\{p_{c}\right\}_{c \in \Omega_{h}}$ be the steady-state solution of the NFV scheme for the pressure formulation. Furthermore, let the relative permeability function satisfy condition (2) and the reconstructed pressure function satisfy conditions (23). Finally, let the permeability tensor $\mathbf{K}$ be constant in each soil. Then, the discrete pressure solution does not have extrema in cells which do not have common edges with both the outside boundary and the boundaries between homogeneous soils.

Proof. The proof is based on the contradictory assumption. Let us assume that there is an extremum, e.g. the maximum, in cell $c$ that is located strictly inside a homogeneous soil:

$$
p_{c} \geqslant p_{c^{\prime}}
$$


for any neighbor cell $c^{\prime}$ and $p_{c}>p_{c^{\prime \prime}}$ for at least one cell $c^{\prime \prime}$. The relative permeability $k_{e}$ is defined through the linear reconstruction and slope-limiting procedures described in Section 3.6. Since $p_{c}$ is the local maximum, then the slope-limiting conditions (23) lead to a constant reconstruction of the pressure inside cell $c$. Moreover, since the relative permeability is the non-decreasing function of $p$, we have $k_{e} \leqslant k_{c}$ for all $e \in \partial c$.

Let us split fluxes in cell $c$ into outward and inward fluxes. For the outward fluxes we have $k_{e}=k_{c}$, since the relative permeability is calculated based on the upwind strategy. Since the inward fluxes are negative, we can bound the mass balance equation as follows:

$$
0=\frac{\rho}{\mu} \sum_{e}^{\text {in }} k_{e} \widetilde{q}_{e}^{h}+\frac{\rho}{\mu} \sum_{e}^{\text {out }} k_{e} \widetilde{q}_{e}^{h} \geqslant \frac{\rho}{\mu} \sum_{e}^{\text {in }} k_{c} \widetilde{q}_{e}^{h}+\frac{\rho}{\mu} \sum_{e}^{\text {out }} k_{c} \widetilde{q}_{e}^{h}=\frac{\rho}{\mu} k_{c} \sum_{e \in \partial c} \widetilde{q}_{e}^{h} .
$$

By our assumption, all neighbors of $c$ belong to the same soil and have the same absolute permeability. Using the definition of fluxes in Section 3, we have

$$
0 \geqslant \sum_{e \in \partial c} \widetilde{q}_{e}^{h}=\sum_{e \in \partial c} d_{e}\left(p_{c}-p_{c^{\prime}}\right)-\rho \sum_{e \in \partial c}\left(\mathbf{K}_{c} \mathbf{g}\right) \cdot \mathbf{n}_{c, e},
$$

where factor $d_{e}$ absorbs both the absolute permeability and local mesh geometry. Recall that $d_{e}$ is a positive number; hence, the first sum in the right-hand side is strictly positive. The second sum is zero. We have a contradiction. The case of a local pressure minimum is treated similarly.

\section{Nonlinear solver}

Let vector $h^{n}=\left\{h_{c}^{n}\right\}_{c \in \Omega_{h}}$ be a cell-centered approximation of the head function at time $t^{n}$. To evolve this approximation to the next time step $t^{n+1}=t^{n}+\Delta t$, we discretize the time derivative in equation (1) using the backward Euler scheme. Since $\rho$ is constant, we obtain the following nonlinear equation in cell $c$ :

$$
\rho \phi_{c} \frac{s_{c}^{n+1}\left(h_{c}^{n+1}\right)-s_{c}^{n}\left(h_{c}^{n}\right)}{\Delta t}+\sum_{e \in \partial c} \rho q_{e}^{n+1}\left(h^{n+1}\right)=0
$$

where $q_{e}^{n+1}\left(h^{n+1}\right)$ is the Darcy flux on edge $e$. Equations (27) for all mesh cells can be written as the system of nonlinear algebraic equations:

$$
\mathcal{F}\left(h^{n+1}\right)=0 \text {. }
$$

To find a solution to (28), we employ the Anderson Acceleration (AA) method [3, 38]. The performance of this method hinges on the contraction property of functional $\mathcal{F}$. We improve the contraction property by employing a preconditioned version of the AA method. In other words, we apply it to a preconditioned nonlinear equation:

$$
\mathcal{G}\left(h^{n+1}\right)=0, \quad \mathcal{G}\left(h^{n+1}\right)=\mathbb{P}^{-1} \mathcal{F}\left(h^{n+1}\right),
$$

where preconditioner $\mathbb{P}$ depends typically on a recent available approximation, $h^{n, s}$, of head $h^{n+1}$. It is known that the closer (in some metric) $\mathbb{P}\left(h^{n, s}\right)$ to the Jacobian matrix of $\mathcal{F}\left(h^{n, s}\right)$, the faster convergence rate of the AA method could be. For the considered NFV schemes, the functional $\mathcal{F}$ can be written as the sum of two terms:

$$
\mathcal{F}\left(h^{n+1}\right)=\mathcal{A}\left(h^{n+1}\right)+\mathbb{D}\left(h^{n+1}\right) h^{n+1},
$$


where $\mathcal{A}\left(h^{n+1}\right)$ is the accumulation term and $\mathbb{D}\left(h^{n+1}\right)$ is the matrix operator representing the approximation of diffusion term $-\operatorname{div}(\rho \mathbf{q})$. While the Jacobian matrix of the accumulation term can be easily computed for a given vector $h^{n, s}$,

$$
\mathbb{J}_{A}=\operatorname{diag}\left\{\frac{\rho \phi_{c}}{\Delta t} \frac{\partial s}{\partial h}\left(h_{c}^{n, s}\right)\right\}_{c \in \Omega_{h}},
$$

derivation of the Jacobian matrix, $\mathbb{J}_{D}$, of the diffusion term is challenging due to nonlinear dependencies hidden in the flux stencil and lack of continuity in the slope limiting algorithm. One standard approach to tackle this problem is to replace the Jacobian matrix with $\mathbb{D}\left(h^{n, s}\right)$ which leads to preconditioner $\mathbb{P}_{D}=\mathbb{J}_{A}+\mathbb{D}$. We use subscript ' $D$ ' to stress the fact that properties of this preconditioner depend mainly on properties of matrix $\mathbb{D}$.

Recently, the new approach for preconditioning Jacobian-Free solvers has been proposed in [21]. This approach defines preconditioner $\mathbb{P}_{L}$ via discretization of the continuous Jacobian of the original PDE which leads to

$$
\mathbb{P}_{L}=\mathbb{J}_{A}+\mathbb{D}+\mathbb{C}
$$

where $\mathbb{C}$ is the correction term which captures nonlinearity of the relative permeability $k_{r}$, see [21] for more details. We use subscript ' $L$ ' to stress the fact that this preconditioner is based on a linearization of the original PDE.

The AA method (see Algorithm 1) computes next solution increment $\Delta h^{n, s+1}=h^{n, s+1}-h^{n, s}$ (with $h^{n}=h^{n, 0}$ and $h^{n+1}=h^{n, s_{\star}+1}$ ) using at most $m \geqslant 1$ recent iterates, where $m$ is a parameter of the method. Let us define vectors $\Delta g^{n, s}=g^{n, s+1}-g^{n, s}$ where $g^{n, s}=\mathcal{G}\left(h^{n, s}\right)$. Let us also define two matrices

$$
\mathbb{G}^{n, s}=\left(\Delta g^{n, s-m_{s}}, \ldots, \Delta g^{n, s-1}\right) \quad \text { and } \quad \mathbb{H}^{n, s}=\left(\Delta h^{n, s-m_{s}}, \ldots, \Delta h^{n, s-1}\right),
$$

where $m_{s}=\min (m, s)$.

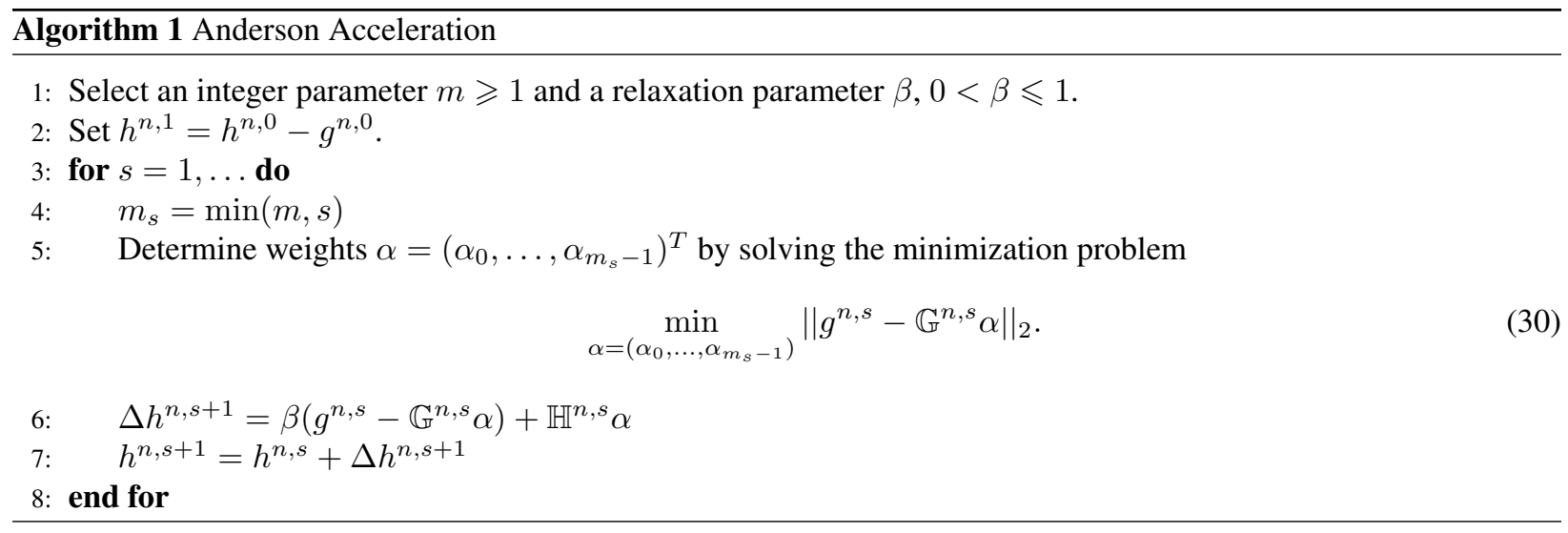

Algorithm 1 requires trivial changes to be adjusted to the pressure formulation of Richards' equation. Hereafter, with a minor abuse of notation, we shall use the same symbol for the discrete nonlinear functional and write discrete equations as $\mathcal{F}\left(p^{n+1}\right)=0$.

\section{Convergence study using 1D infiltration problem (head formulation)}

In this section we study the approximation properties of the proposed NFV schemes using the analytic steady-state solution of Richards' equation presented in [27]. Since the main focus is on scheme accuracy, our choice of parameters does not reflect real physical quantities. 
We consider the rectangular computational domain $\Omega=[0,2] \times[0,10]$ and set $g=2, \rho=\mu=1$, and $p_{\text {atm }}=0$. The computational domain is divided into equal subdomains $\Omega_{i}, i=1,2$, as follows: $\Omega_{1}=[0,2] \times[10,5]$ and $\Omega_{2}=[0,2] \times[5,0]$. The permeability tensor is a constant function in each subdomain, $\mathbf{K}(x, z)=0.5$ in $\Omega_{1}$ and $\mathbf{K}(x, z)=2$ in $\Omega_{2}$. The no-flow boundary condition is imposed on the vertical boundaries $\Gamma_{N}$, see Fig. 2 . We set $h=10$ on the top boundary and $h=0$ on the bottom one. The derivations in [27] use the following model for the relative permeability:

$$
k\left(p_{c}\right)=\frac{1}{1+p_{c}^{2}},
$$

where $p_{c}$ is the capillary pressure. The analytic solution (see Fig.3) is given by

$$
\begin{cases}p_{c}(x, z)=\Phi_{1} \tan \left(\frac{\Phi_{1} c_{r}(10-z)}{k_{1}}\right) & \text { if } z>0.5, \\ p_{c}(x, z)=-\Phi_{2} \tanh \left(\frac{c_{r} \Phi_{2}(5-z)}{k_{2}}-\operatorname{arctanh}\left(\frac{\Phi_{1}}{\Phi_{2}} \tan \left(5 \frac{c_{r} \Phi_{1}}{k_{1}}\right)\right)\right) & \text { if } z<0.5,\end{cases}
$$

where $c_{r}=1.02160895463, \Phi_{1}=\sqrt{1-2 \frac{K_{1}}{c_{r}}}$, and $\Phi_{2}=\sqrt{2 \frac{K_{2}}{c_{r}}-1}$.

The convergences studies are performed on a sequence of distorted quadrilateral meshes like that shown on the right panel in Fig. 2. Such meshes are very challenging for any numerical scheme since no error cancellation is possible due to huge amount of random noise in the position of mesh vertices. The discrete $L^{2}$-norm of the pressure error is presented in Table 1. In the third and fifth column of Table 1 logarithm of ratio of error norms on consecutive meshes is shown. The numbers confirm our expectation that the first-order (resp., the second-order) reconstruction of the pressure leads to the first-order (resp., the second-order) convergence rate on distorted meshes. Note that the low-order scheme saturates to the optimal convergence rate more faster than the second-order scheme. We think that this effect is due to our limiter or, more precisely, due to the size of set $\Sigma_{c}$ that includes all neighboors of cell $c$. In addition, we note that the discrete head on all meshes in this set satisfies the discrete maximum principle. Also, no local extrema in the discrete pressure were observed inside subdomains $\Omega_{i}$, even on coarse meshes.

\begin{tabular}{r||c|c||c|c}
\multicolumn{1}{c||}{} & \multicolumn{4}{c}{ discrete $L^{2}$-norm of pressure error } \\
\hline mesh & NFV I & $\log _{2}$ (ratio) & NFV II & $\log _{2}$ (ratio) \\
\hline $8 \times 2$ & $1.93 \mathrm{e}-01$ & - & $8.04 \mathrm{e}-02$ & - \\
$16 \times 4$ & $1.19 \mathrm{e}-01$ & 0.70 & $3.34 \mathrm{e}-02$ & 1.27 \\
$32 \times 8$ & $6.74 \mathrm{e}-02$ & 0.82 & $1.25 \mathrm{e}-02$ & 1.42 \\
$64 \times 16$ & $3.71 \mathrm{e}-02$ & 0.86 & $4.34 \mathrm{e}-03$ & 1.53 \\
$128 \times 32$ & $2.00 \mathrm{e}-02$ & 0.90 & $1.44 \mathrm{e}-03$ & 1.59 \\
$256 \times 64$ & $1.05 \mathrm{e}-02$ & 0.93 & $4.35 \mathrm{e}-04$ & 1.73 \\
$512 \times 128$ & $5.43 \mathrm{e}-03$ & 0.95 & $1.21 \mathrm{e}-04$ & 1.85
\end{tabular}

Table 1: Convergence results for analytic solution (31) for the first- and second-order NFV schemes.

\section{Infiltration into partially saturated soils}

In this section, we model the infiltration process into a partially saturated two-dimensional domain which has a layered structure shown schematically on the left panel in Fig. 4. The width of the domain is $200 \mathrm{~m}$ and the maximum height is $125 \mathrm{~m}$. In all experiments, we impose the no-flow boundary conditions on the lateral boundaries and the 

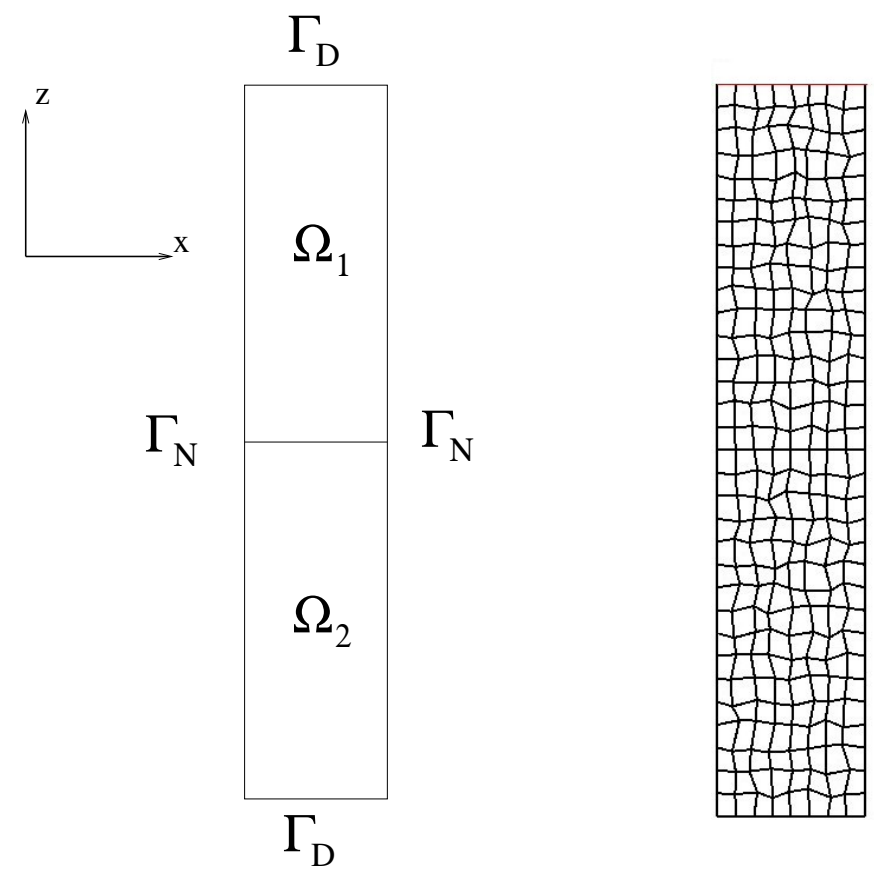

Figure 2: A sketch of the model (left panel) and a distorted quadrilateral mesh used in the convergence analysis (right panel).
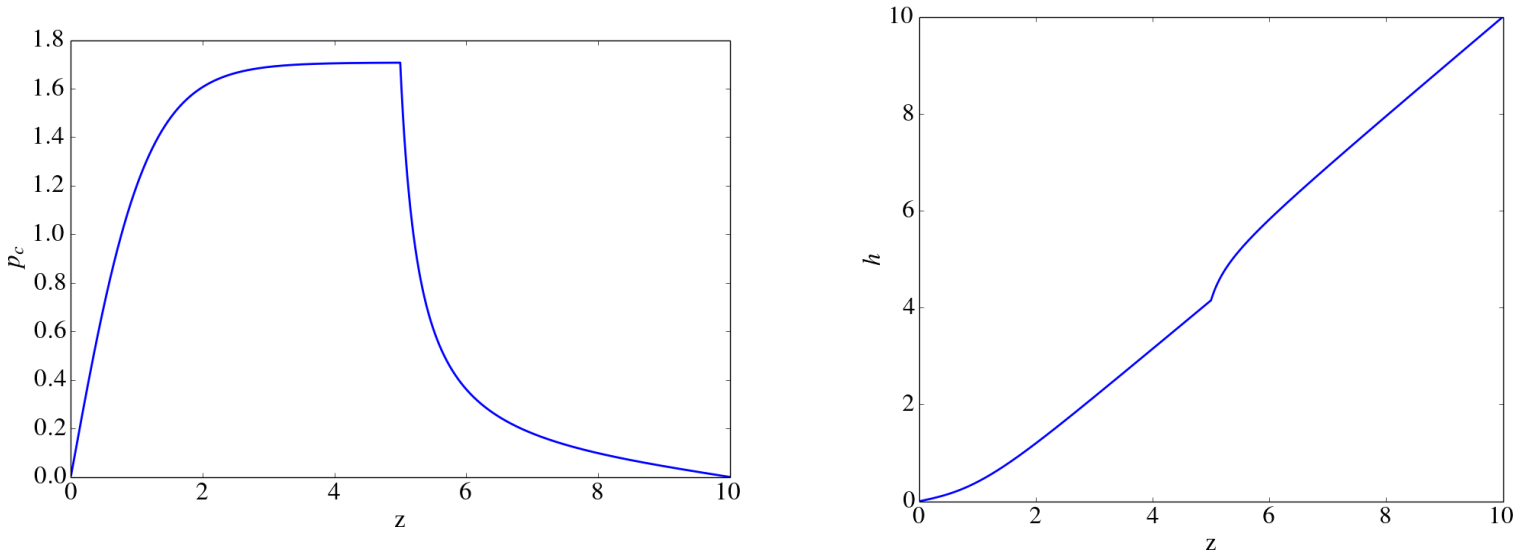

Figure 3: The piecewise monotone analytic pressure (left panel) and globally monotone hydraulic head (right panel). 

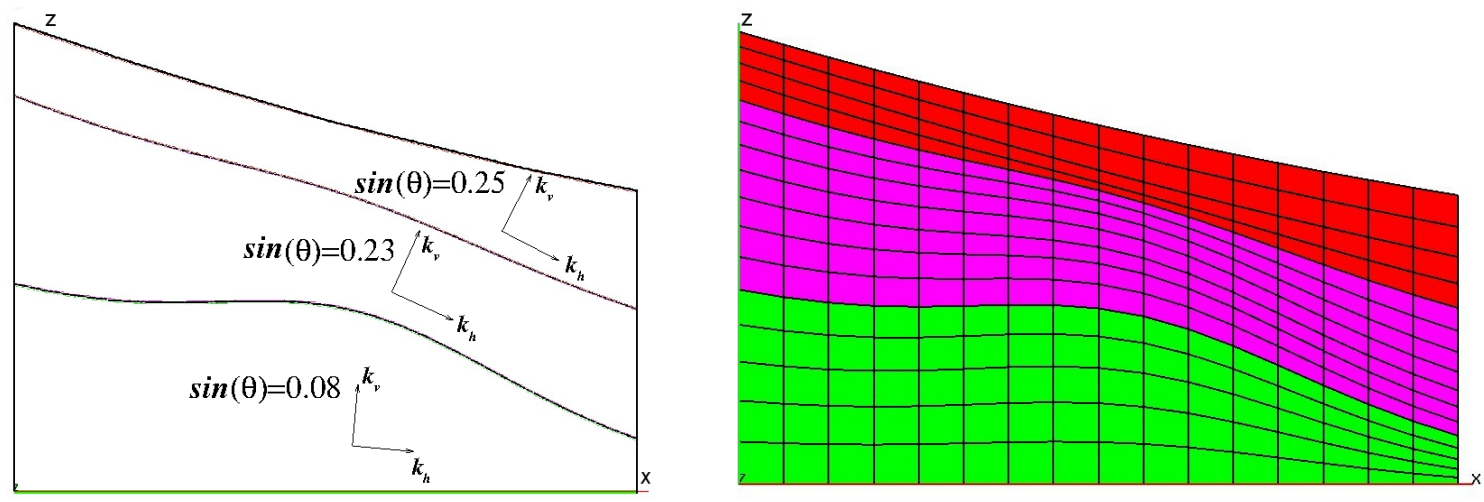

Figure 4: A sketch of the model (left panel) and fitted mesh (right panel).

homogeneous Dirichlet boundary condition $\left(p=p_{a t m}=101325 \mathrm{~Pa}\right)$ on the bottom boundary. In each layer, the absolute permeability is given by a constant full anisotropic permeability tensor:

$$
\mathbf{K}=R(-\theta)\left(\begin{array}{cc}
K_{h} & 0 \\
0 & K_{v}
\end{array}\right) R(\theta), \quad R(\theta)=\left(\begin{array}{rr}
\cos \theta & \sin \theta \\
-\sin \theta & \cos \theta
\end{array}\right),
$$

where $K_{h}$ and $K_{v}$ are permeabilities along averaged principal directions of a layer and $\theta$ is the constant angle between the principal directions and Cartesian axes. Constant tensors were selected to verify the assertion of Theorem 3.2. We use the van Genuchten-Mualem water retention model (3) to define the relative permeability.

To generate a realistic structure of subsurface layers we use the following mapping from the unit square to computational domain $\Omega$ :

$$
\begin{array}{ll}
\hat{x}:=200 x & (x, z) \in[0,1] \times[0,1], \\
\hat{z}:=100 z+6.5 z(14-x-10 z)(1-2 x)+100 z\left(1-\frac{4}{3} z\right) e^{-(10 x-5.5)^{2} / 10} & (x, z) \in[0,1] \times[0,0.5], \\
\hat{z}:=100 z+6.5 z(14-x-10 z)(1-2 x)+80 z\left(1-\frac{20}{17} z\right) e^{-(10 x-5.5)^{2} / 10} & (x, z) \in[0,1] \times(0.5,0.85], \\
\hat{z}:=100 z+6.5 z(14-x-10 z)(1-2 x) & (x, z) \in[0,1] \times(0.85,1] .
\end{array}
$$

First, in the unit square we define three subdomains as follows: the bottom layer $-(x, z) \in[0,1] \times[0,0.325)$, the middle layer - $(x, z) \in[0,1] \times[0.325,0.75)$ and the top layer - $(x, z) \in[0,1] \times[0.75,1]$. Then a mesh fitted to interfaces between different soils is obtained by mapping an orthogonal mesh (on the unit square). The fitted mesh and transformed subdomains which represent three different soil layers are illustrated on the right panel in Fig. 4.

In this study we consider several mesh resolutions with the coarsest mesh having $32 \times 32$ cells and the finest one having $128 \times 128$ cells. The coarsest mesh is challenging for many numerical schemes to satisfy the discrete maximum principles. The finest mesh shows clear advantage of the second-order NFV scheme.

\subsection{Phase I: calculation of a steady-state solution}

Due to lack of a reasonable initial guess, a physically meaningful solution must be computed at the beginning of a simulation. This phase of a simulation is somewhat special and employs various acceleration techniques. The most popular approach is to calculate a steady-state solution by evolving the system during a long period of time. We prescribe a constant mass flux on the top boundary of $\Omega$ with the value of $1.1071 \cdot 10^{-7}\left[\mathrm{~kg} \cdot \mathrm{m} \cdot \mathrm{s}^{-1}\right]$ and evolve the system for 2000 years. The final solution becomes the initial guess for the transient phase. 
At each time step we solve the system of nonlinear algebraic equations (1) using the AA method described in Section 4. The convergence criteria for the AA method effect not only its performance but also properties of the resulting numerical solution. Although in many applications the criteria are based on the $L^{2}$-norm of the nonlinear residual, for the infiltration problem such criteria are typically too weak. Importance of local solution dynamics requires a stronger convergence criterion that is the combination of the infinity norms of the normalized nonlinear residual and of the solution increment (hereafter, we drop the time superscript and keep only the iteration index of the nonlinear solver). Depending on the formulation (head or pressure), we use either

$$
e_{h}^{s}:=\max \left(\frac{\left\|\Delta h^{s}\right\|_{\infty}}{h_{\max }}, \frac{\left\|\mathcal{F}\left(h^{s}\right)\right\|_{\infty}}{\left\|\mathcal{F}\left(h^{0}\right)\right\|_{\infty}}\right) \leqslant \varepsilon_{n o n} \quad \text { or } \quad e_{p}^{s}:=\max \left(\frac{\left\|\Delta p^{s}\right\|_{\infty}}{P_{a t m}}, \frac{\left\|\mathcal{F}\left(p_{s}\right)\right\|_{\infty}}{\left\|\mathcal{F}\left(p_{0}\right)\right\|_{\infty}}\right) \leqslant \varepsilon_{n o n},
$$

where $h_{\max }=125$. The nonlinear tolerance is set to $\varepsilon_{n o n}=10^{-5}$ in all tests. The initial guess is based on the clipped hydrostatic pressure:

$$
h(x, z)=\max \left\{\frac{p_{\text {clip }}-p_{a t m}}{\rho g}+z, 0\right\} \quad \text { or } \quad p(x, z)=\max \left\{p_{a t m}-\rho g z, p_{c l i p}\right\},
$$

which limits the initial pressure value by $p_{\text {clip }}$ to avoid extremely dry (non-physical) regions at the beginning of calculations that cause problems for both the nonlinear solver and preconditioners. This clipping of the hydrostatic pressure improves convergence of the nonlinear solver since it eliminates large solution gradients when a wetting front propagates inside the domain. The value of $p_{c l i p}$ which provides the most effective performance of the nonlinear solver is problem dependent. In the most of the presented numerical tests, we set $p_{\text {clip }}=0.5 p_{\text {atm }}$.

Efficient simulation requires also a variable time stepping strategy. Here and in the subsequent experiments, we apply a heuristic time stepping strategy based on the current number of nonlinear iterations, $n_{n l}$. If $\Delta t_{n}$ is the current time step, the next time step is calculated as follows:

$$
\Delta t_{n+1}=\xi \Delta t_{n} \quad \text { where } \quad \xi=\left\{\begin{array}{lll}
1.25 & \text { if } n_{n l} \leqslant n_{\min } \\
1 & \text { if } n_{\min }<n_{n l} \leqslant n_{\max } \\
0.5 & \text { if } \quad n_{\max }<n_{n l}
\end{array}\right.
$$

and parameters $n_{\min }$ and $n_{\max }$ are set as follows: $n_{\min }=10, n_{\max }=15$.

\subsubsection{Performance of the AA method for different pressure reconstruction schemes}

Let us consider different sets of parameters for the van Genuchten-Mualem water retention model (WRM) which affect the nature of nonlinearity for the problem. Some researchers stress out the fact that the relative permeability curve has different properties for $n \geqslant 2$ and $n<2$, [25]. If $n<2$, the derivative of $k\left(p_{c}\right)$ is discontinuous at $p_{c}=0$ which may affect convergence of nonlinear solvers. In practice, this problem is mitigated by regularizing the curve around $p_{c}=0$. Overall, we consider six sets of parameters that are summarized in Table 2. Equal number of sets correspond to smooth and non-smooth curves. These parameter values are taken from a number of sources [32, 37] and cover wide range of possible smooth and non-smooth water retention curves.

Different properties of the relative permeability curves lead to very different profiles of the Darcy velocity and different characteristic times for the steady-state and transient simulations. To have similar characteristic times, we set the anisotropy ratio, $K_{h} / K_{v}$, to 10 for non-smooth $k_{r}$ and to 2 otherwise. The values of permeabilities along principal direction are shown in Table 3.

The average number of nonlinear iterations per time step and the total number of nonlinear iterations for the overall simulation are presented in Table 4 for the head formulation and in Table 5 for the pressure formulation. The number of nonlinear iterations is computed as the sum of nonlinear iterations on all time steps including the failed steps (when 


\begin{tabular}{c|c|c||c|c|c}
\multicolumn{3}{c||}{ Non-smooth $k_{r}$} & \multicolumn{3}{c}{ Smooth $k_{r}$} \\
\hline WRM \# & $n$ & $\alpha$ & WRM \# & $n$ & $\alpha$ \\
\hline 1 & 1.43 & $2.0674 \mathrm{e}-4$ & 4 & 3.18 & $3.5959 \mathrm{e}-4$ \\
2 & 1.30 & $1.9467 \mathrm{e}-4$ & 5 & 5.00 & $1.0000 \mathrm{e}-4$ \\
3 & 1.27 & $2.0260 \mathrm{e}-4$ & 6 & 2.76 & $2.0431 \mathrm{e}-5$
\end{tabular}

Table 2: Different sets of parameters for the van Genuchten-Mualem water retention model.

\begin{tabular}{c|c|c||c|c} 
& \multicolumn{2}{|c|}{ Non-smooth $k_{r}$} & \multicolumn{2}{c}{ Smooth $k_{r}$} \\
& $K_{h}\left[\mathrm{~m}^{-2}\right]$ & $K_{v}\left[\mathrm{~m}^{-2}\right]$ & $K_{h}\left[\mathrm{~m}^{-2}\right]$ & $K_{v}\left[\mathrm{~m}^{-2}\right]$ \\
\hline Top layer & $1 \mathrm{e}-10$ & $1 \mathrm{e}-11$ & $1 \mathrm{e}-10$ & $5 \mathrm{e}-11$ \\
Middle layer & $1 \mathrm{e}-11$ & $1 \mathrm{e}-12$ & $1 \mathrm{e}-11$ & $5 \mathrm{e}-12$ \\
Bottom layer & $1 \mathrm{e}-10$ & $1 \mathrm{e}-11$ & $1 \mathrm{e}-10$ & $5 \mathrm{e}-11$
\end{tabular}

Table 3: Horizontal and vertical permeabilities in three different layers.

the solver did not converge in $n_{\max }$ iterations or diverged). Our experiments demonstrate that the total number of nonlinear iterations is a good measure to evaluate the overall computational cost since it is proportional to the total CPU time.

For non-smooth cases (WRM 1-3), we observe that the second-order reconstruction does not harm the performance of the AA method. In some cases, the number of nonlinear iterations even reduces. For smooth cases (WRM 4-6), especially for WRM 4-5, the difference in the number of iterations between NFV I and NFV II schemes is more profound. For the cases WRM 4-5, the soils are more permeable than in the other cases. This results in a boundary layer near the right (no-flow) boundary of the domain, see Fig 5, which slows convergence of the AA method. The difference is mitigated with mesh refinement.

In all tests the nonlinear convergence for the pressure formulation was slower and sometimes significantly slower than for the head formulation. The variable time stepping strategy was designed to reduce the number of failed time steps. This strategy does not allow the time step to grow when the nonlinear solver exhibits slow convergence. This leads to the fact that the average number of iterations per time step is similar in both formulations (close to the limit value $n_{\min }$ ), but the total number of time steps is larger for the pressure formulation.

It was noticed in [19] that when the diffusive and gravity fluxes are upwinded using the same upwind strategy, the resulting functional may become highly nonlinear. It is not the issue for the head formulation, since there are no gravity fluxes. The multi-point nature of our diffusive flux discretization requires serious revision of the hybrid upwind strategy proposed in [19] and it will be the topic of future research. Since the head formulation demonstrates faster convergence, we consider only this formulation in the subsequent test cases.

It is important to note that the average number of nonlinear iteration per time step is insensitive to both the mesh refinement and choice of the WRM. This fact demonstrates the effectiveness of our variable time stepping algorithm.

In Fig. 5 we present steady-state pressure profiles computed by the TPFA scheme and the NFV I and NFV II schemes for WRM 1 and WRM 4. Note that on such a fine mesh, it is difficult to determine the difference between the NFV I and NFV II schemes in the "eyeball-norm". However, these figures demonstrate clearly the difference between the schemes which have the formal order of convergence (NFV schemes) and the non-convergent scheme (TPFA). This difference is more profound for the non-smooth case WRM 1.

The discrete head solutions provided by three schemes satisfy the discrete maximum principle on all meshes, but only the NFV I and NFV II lead to accurate solution approximation. Although the pressure formulation guarantees the discrete maximum principle only for the aqueous pressure and the head formulation - only for the hydraulic head, in all presented test cases we observed that both maximum principles hold. 


\begin{tabular}{c|cc|cl|ll} 
& \multicolumn{2}{|c|}{ mesh 32x32 } & \multicolumn{2}{c|}{ mesh 64x64 } & \multicolumn{2}{c}{ mesh 128x128 } \\
\hline WRM & NFV I & NFV II & NFV I & \multicolumn{1}{c}{ NFV II } & NFV I & \multicolumn{1}{c}{ NFV II } \\
\hline 1 & $3140(8.8)$ & $3475(9.3)$ & $6227(9.7)$ & $6601(10.2)$ & $8107(10.3)$ & $7701(10.6)$ \\
2 & $3742(9.1)$ & $4566(9.0)$ & $7379(9.7)$ & $7500(9.8)$ & $9421(10.3)$ & $9371(10.4)$ \\
3 & $3872(9.1)$ & $4190(9.3)$ & $7103(9.7)$ & $7532(9.9)$ & $9336(10.5)$ & $9166(10.6)$ \\
\hline 4 & $2905(8.5)$ & $5629(9.5)$ & $4777(9.5)$ & $8565(10.4)$ & $5277(10.5)$ & $9760(11.5)$ \\
5 & $2038(8.2)$ & $5130(9.0)$ & $3220(8.9)$ & $6644(9.5)$ & $4042(8.9)$ & $7040(9.7)$ \\
6 & $2336(8.2)$ & $2495(8.5)$ & $5209(9.1)$ & $4978(9.2)$ & $10701(9.7)$ & $10466(9.8)$
\end{tabular}

Table 4: Head formulation: the total number of nonlinear iterations (the average number of nonlinear iteration per time step) required to compute the steady-state solution at $\mathrm{T}=2000 \mathrm{y}$.

\begin{tabular}{c|cc|cl|ll} 
& \multicolumn{2}{|c|}{ mesh 32x32 } & \multicolumn{2}{c|}{ mesh 64x64 } & \multicolumn{2}{c}{ mesh 128x128 } \\
\hline WRM & NFV I & NFV II & \multicolumn{1}{|c}{ NFV I } & NFV II & \multicolumn{1}{c}{ NFV I } & NFV II \\
\hline 1 & $4074(9.9)$ & $4418(9.0)$ & $10797(10.2)$ & $11151(10.3)$ & $24747(10.7)$ & $25308(11.0)$ \\
2 & $4480(9.9)$ & $4982(9.9)$ & $11796(10.3)$ & $12489(10.3)$ & $25369(10.9)$ & $26486(10.9)$ \\
3 & $4593(9.8)$ & $5001(9.8)$ & $11434(10.4)$ & $12488(10.4)$ & $26854(10.6)$ & $27348(10.8)$ \\
\hline 4 & $5018(9.1)$ & $7418(9.2)$ & $10470(9.2)$ & $18954(9.2)$ & $18611(9.1)$ & $30108(9.2)$ \\
5 & $6284(9.2)$ & $8466(9.2)$ & $13291(8.7)$ & $24179(8.8)$ & $23908(9.0)$ & $36405(8.7)$ \\
6 & $3118(9.1)$ & $3376(9.2)$ & $6713(9.6)$ & $7001(9.7)$ & $14879(10.1)$ & $14586(10.2)$
\end{tabular}

Table 5: Pressure formulation: the total number of nonlinear iterations (the average number of nonlinear iteration per time step) required to compute the steady-state solution at $\mathrm{T}=2000 \mathrm{y}$.

\subsubsection{Advanced preconditioner techniques}

In Section 4, we described two preconditioning strategies for the nonlinear solver. In the previous tests, we used preconditioner $\mathbb{P}_{D}$ that ignores nonlinearity of the relative permeability. Here, we compare the efficacy of this preconditioner with the preconditioner proposed recently in [21] which uses a stable discretization of the continuous Jacobian of the original PDE.

Let us repeat some of the above tests using the new preconditioner and the head formulation. More precisely, we recompute the steady-state solutions on the finest $128 \times 128$ mesh for all six water retention models. The results are presented in Table 6. For three non-smooth cases, the improvement is quite moderate and is in the range of $10 \%$. On the other hand, for the smooth cases, we observe more significant speed up. Note that impact of the new preconditioner is stronger in the cases where the difference in the iteration count between the NFV I and NFV II schemes is more distinct.

\begin{tabular}{c|ll|ll} 
& \multicolumn{2}{|c|}{ NFV I } & \multicolumn{2}{c}{ NFV II } \\
\hline WRM & \multicolumn{1}{|c}{$\mathbb{P}_{D}$} & \multicolumn{1}{c}{$\mathbb{P}_{L}$} & \multicolumn{1}{c}{$\mathbb{P}_{D}$} & $\mathbb{P}_{L}$ \\
\hline 1 & $8107(10.3)$ & $7581(10.5)$ & $7701(10.6)$ & $7123(10.5)$ \\
2 & $9421(10.3)$ & $9327(10.2)$ & $9371(10.4)$ & $8569(10.2)$ \\
3 & $9336(10.5)$ & $8702(10.2)$ & $9166(10.6)$ & $8540(10.3)$ \\
\hline 4 & $5277(10.5)$ & $2787(8.8)$ & $9760(11.5)$ & $4139(8.7)$ \\
5 & $4042(8.9)$ & $2473(8.1)$ & $7040(9.7)$ & $2688(8.5)$ \\
6 & $10701(9.7)$ & $9450(10.4)$ & $10466(9.8)$ & $9757(9.8)$
\end{tabular}

Table 6: Comparison of two preconditioning strategies: the total number of nonlinear iterations (the average number of nonlinear iterations per time step) required to compute the steady-state solution at $\mathrm{T}=2000 \mathrm{y}$ on the finest mesh. 

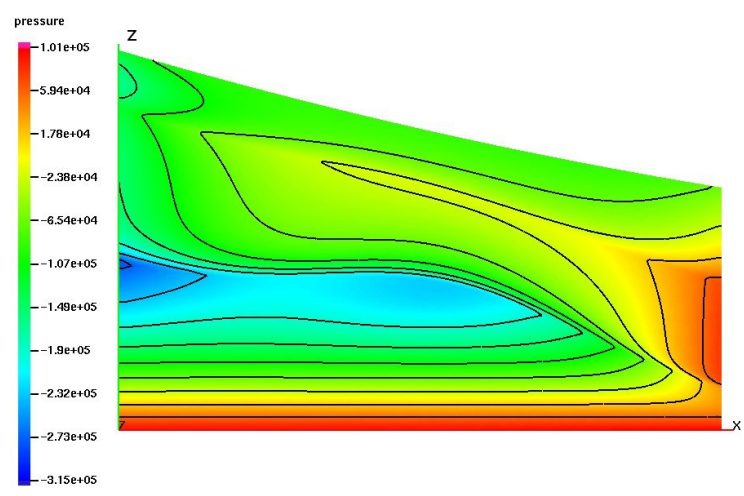

TPFA

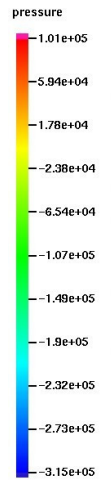

pressure
$-1.01 \mathrm{e}+05$
$-5.94 \mathrm{e}+04$
$-1.78 \mathrm{e}+04$
$--2.38 \mathrm{e}+04$
$--6.54 \mathrm{e}+04$
$-1.07 \mathrm{e}+05$
$-1.99 \mathrm{e}+05$
$-1.9 \mathrm{e}+05$
$-2.32 \mathrm{e}+05$
$-2.73 \mathrm{e}+05$
$-3.15 \mathrm{e}+05$

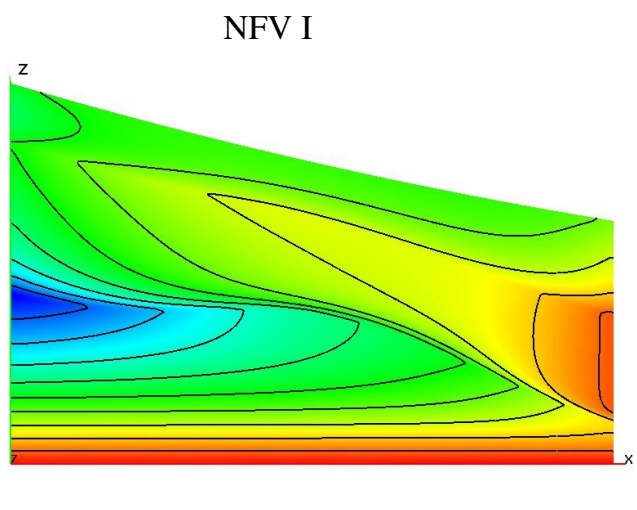

NFV II

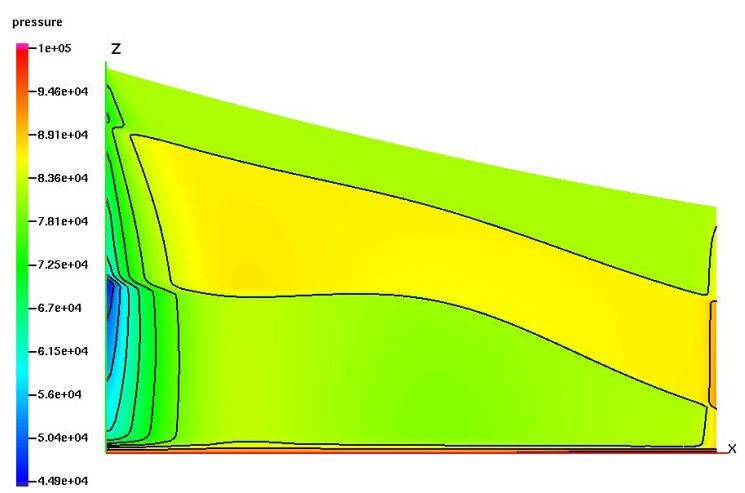

TPFA
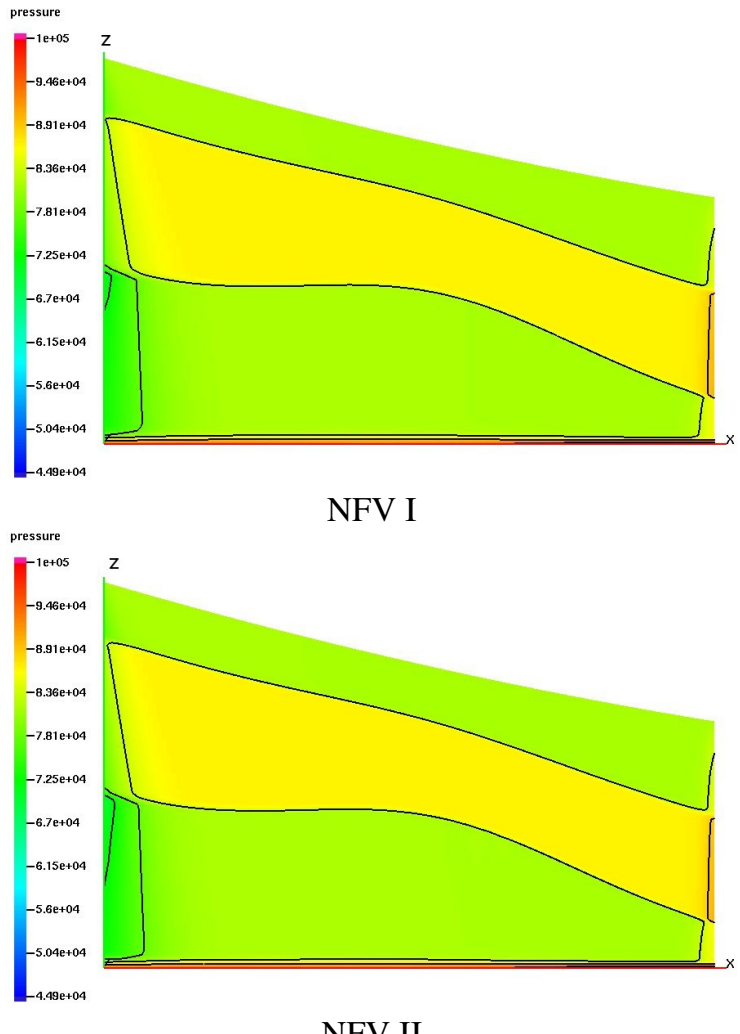

NFV II

Figure 5: The steady-state pressure profiles computed on mesh $128 \times 128$ for the WRM1 (left panel) and WRM 4 (right panel).

\subsection{Phase II: calculation of a transient solution}

The difference between the first- and second-order schemes is more noticeable for transient problems. For transient tests, we consider only one water retention model WRM 1 . The initial head is computed as described in the previous section. On the top boundary $\Gamma_{t o p}$ of $\partial \Omega$, we define the recharge subdomain $\Gamma_{r}=\left\{(x, y) \in \Gamma_{\text {top }}: x \in[0,40]\right\}$. 
At the beginning of the transient simulation, the infiltration rate is set to $1.1071 \mathrm{e}-4\left[\mathrm{~kg} \cdot \mathrm{m} \cdot \mathrm{s}^{-1}\right]$ for $\Gamma_{r}$ and to the background value $1.1071 \mathrm{e}-7\left[\mathrm{~kg} \cdot \mathrm{m} \cdot \mathrm{s}^{-1}\right.$ ] for the rest of the top boundary. After 1 year, the recharge process is turned off and the boundary flux is set to the background value. The transient simulation runs for another 4 years, driven mostly by gravity.

\begin{tabular}{c|c|c|c|c} 
& \multicolumn{2}{|c|}{$\mathbb{P}_{D}$} & \multicolumn{2}{|c}{$\mathbb{P}_{L}$} \\
\hline mesh & NFV I & NFV II & NFV I & NFV II \\
\hline $32 \times 32$ & $1237(5.30)$ & $1675(5.93)$ & $796(3.77)$ & $839(4.30)$ \\
$64 \times 64$ & $2115(6.73)$ & $2600(7.02)$ & $917(4.80)$ & $1265(5.40)$ \\
$128 \times 128$ & $2798(8.06)$ & $3495(7.96)$ & $1253(5.77)$ & $1613(6.58)$
\end{tabular}

Table 7: Total number of nonlinear iterations (the average number of nonlinear iteration per time step) in the transient phase for NFV I and NFV II schemes using different preconditioning approaches.

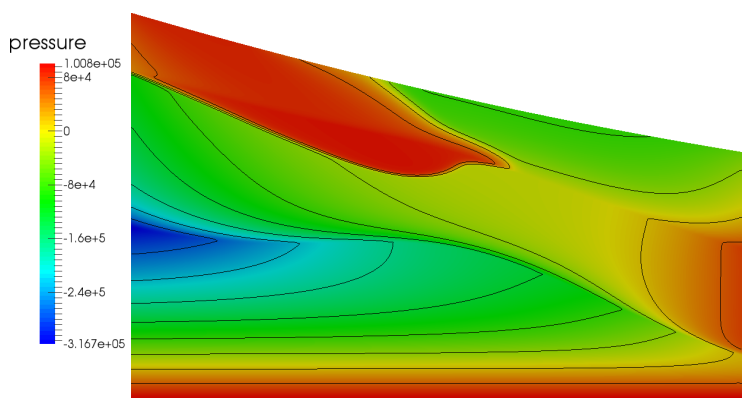

After 1 year

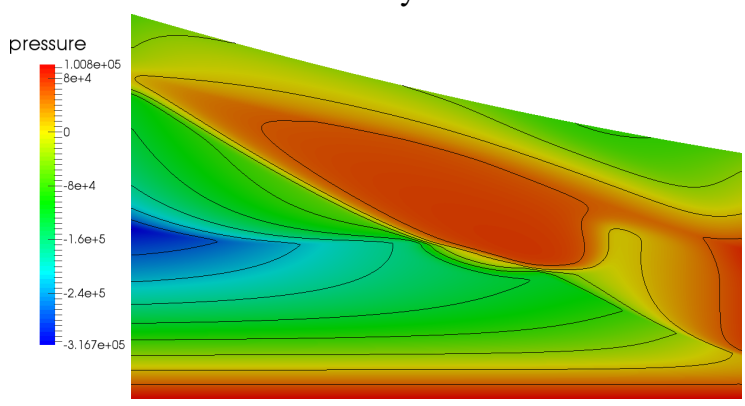

After 5 years

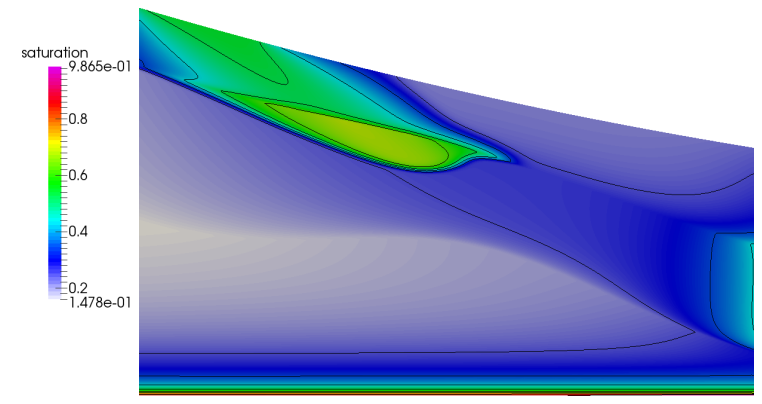

After 1 year

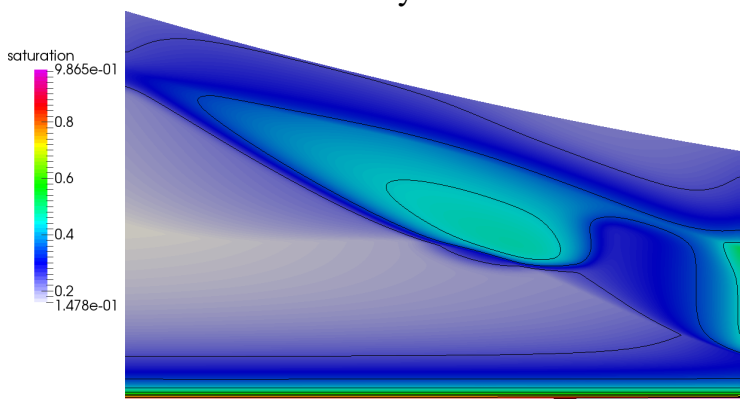

After 5 years

Figure 6: Pressure and saturation profiles for the transient simulation.

To accommodate sharp changes in the boundary conditions, we reduce time step to 1 second every time these changes occur. The decrease in the number of nonlinear iterations per a time step in comparison with the steady-state experiments, see Table 7, is due to this reset and the shorter simulation time. Moreover, in contrast to the steady-state calculation, at every time step in the transient phase, we have a better initial guess which leads to significant reduction of the number of failed time steps. The new preconditioning strategy improves convergence of AA solver in more than two times. We also conclude that the overhead caused by the second-order pressure reconstruction stays in the range of $30 \%$ of the number of nonlinear iterations. The profiles of pressure and saturation are shown in Fig 6.

The difference between the first- and second-order schemes is easier to analyze using one dimensional slices of 


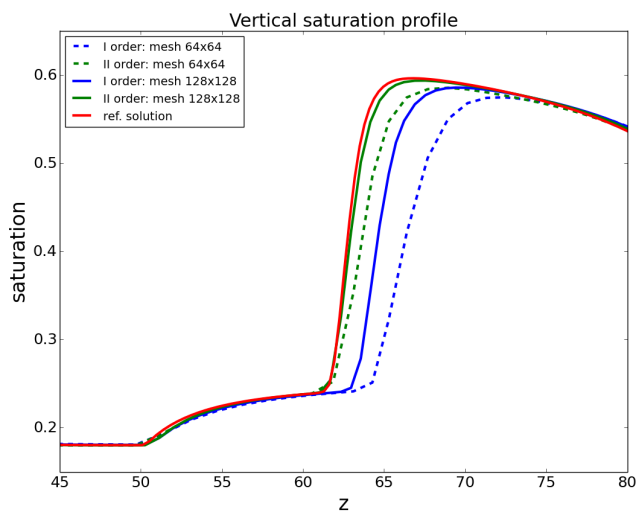

(a) $T=1.5$ years

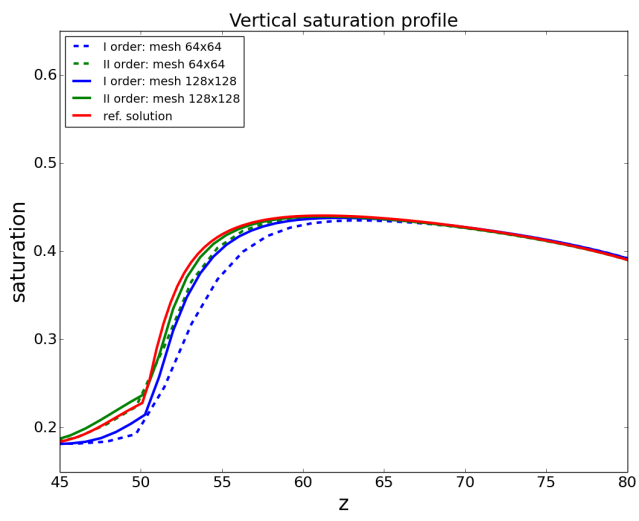

(c) $T=5$ years

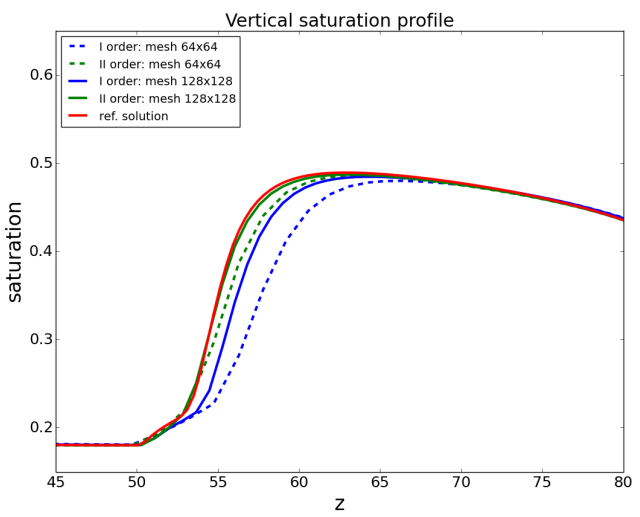

(b) $T=3$ years

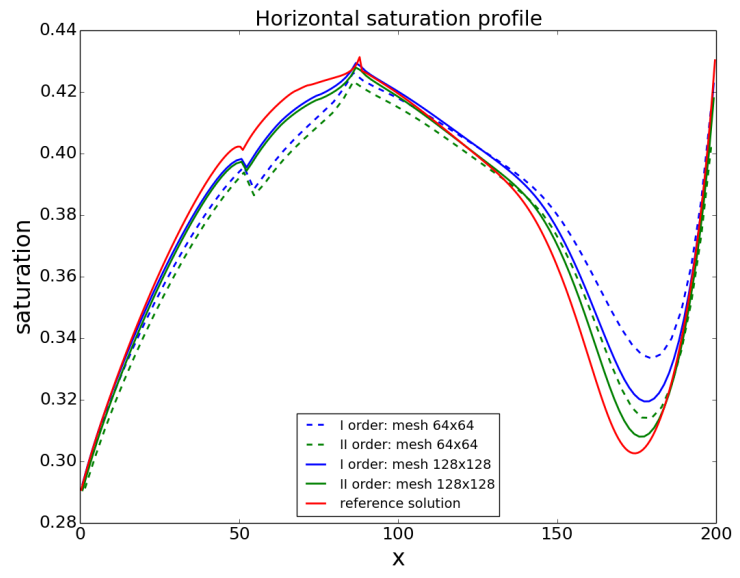

(d) $T=3$ years

Figure 7: Panels (a)-(c): Slices at $x=100 \mathrm{~m}$ of saturation for different times computed on $64 \times 64$ and $128 \times 128$ meshes by NFV I and NFV II scheme in comparison with the reference solution. Panel (d): Slices of saturation along the line connecting points $(0,110)$ and $(200,45)$ at $\mathrm{T}=3 \mathrm{y}$.

the saturation profiles. In Fig. 7 a,b,c, we present slices at $x=100 \mathrm{~m}$. The slices are made for numerical solutions computed on meshes $64 \times 64$ and $128 \times 128$ using different pressure reconstruction methods and compared with the reference solution computed on the mesh $256 \times 256$ using the NFV II scheme. We observe that the position and sharpness of the saturation front is more closer to the reference solution for the second-order scheme than for the first-order one. Even on the coarser mesh, the second-order scheme provides higher accuracy than the first-order scheme on the twice refined mesh which has 4 times more unknowns. Since all presented schemes conserve mass, the slower propagation of the water front in the z-direction is compensated by a wider spread of the water plume in the $\mathrm{x}$-direction. This effect is confirmed by the horizontal cut-lines of the saturation profiles presented in Fig $7 \mathrm{~d}$. In conclusion, the presented results justify the overhead caused by the second-order scheme. 


\section{Conclusion}

We derived two second-order accurate nonlinear finite volume (NFV) schemes on unstructured polygonal meshes for solving Richards' equation in a multi-layered medium written in the head and pressure formulations. These scheme preserve important mathematical properties of the continuous solution. For the head formulation, we proved that the steady-state hydraulic head has no extrema inside the computational domain. For the pressure formulation, if in addition the absolute permeability is constant in each layer, we proved that the aqueous pressure has no extrema inside each layer but not globally in the computational domain.

We demonstrated numerical efficiency of the second-order NFV scheme with a few challenging problems of water infiltration into a dry soil. Propagation of the wetting front was predicted more accurately by this scheme than by the first-order NFV scheme on the twice refined mesh. Since calculation of the Jacobian matrix for the secondorder scheme is prohibitively expensive due to build-in reconstruction and slope limiting algorithms, we compared performance of the conventional diffusion-based preconditioner with the preconditioner introduced recently in [21]. The new preconditioner showed consistent reduction of the computational time, about twice in half of our experiments.

\section{Acknowledgments}

This work was carried out under the auspices of the National Nuclear Security Administration of the U.S. Department of Energy at Los Alamos National Laboratory under Contract No. DE-AC52-06NA25396, the DOE Office of Science Advanced Scientific Computing Research (ASCR) Program in Applied Mathematics and by the Next Generation Ecosystem Experiment (NGEE-Arctic) project. NGEE-Arctic is supported by the Office of Biological and Environmental Research in the DOE Office of Science.

\section{References}

[1] I. Aavatsmark, G.T. Eigestad, B.T. Mallison, and J.M. Nordbotten. A compact multipoint flux approximation method with improved robustness. Numer. Meth. Partial Diff. Eqns., 24(5):1329-1360, 2008.

[2] L. Agelas, R. Eymard, and R. Herbin. A nine-point finite volume scheme for the simulation of diffusion in heterogeneous media. C. R. Acad. Sci. Paris, Ser. I, 347:673-676, 2009.

[3] D. Anderson. Iterative procedures for nonlinear integral equations. J. ACM, 12(4):547-560, 1965.

[4] T. Barth. Aspects of unstructured grids and finite-volume solvers for the euler and navier-stokes equations. AGARD, Rep.787, 1992.

[5] B. Belfort, A. Younes, M. Fahs, and F. Lehmann. On equivalent hydraulic conductivity for oscillation-free solutions of richard's equation. Journal of Hydrology, 505:202-217, 2013.

[6] L. Bergamaschi and M. Putti. Mixed finite elements and newton-type linearizations for the solution of richards' equation. Int. J. Numer. Meth. Eng., 45(8):1025-1046, 1999.

[7] A. Berman and R. J. Plemmons. Nonnegative matrices in the mathematical sciences. Academic Press [Harcourt Brace Jovanovich Publishers], New York, 1979. Computer Science and Applied Mathematics.

[8] M. A. Celia and E. T. Bouloutas. A general mass-conservative numerical solution for the unsaturated flow equation. Water Resour. Res., 26(7):1483-1496, 1990. 
[9] A. Danilov and Y. Vassilevski. A monotone nonlinear finite volume method for diffusion equations on conformal polyhedral meshes. Russian J. Numer. Anal. Math. Modelling, 24(3):207-227, 2009.

[10] M. Dotlic, D. Vidovic, B. Pokorni, M. Pusic, and M. Dimkic. Second-order accurate finite volume method for well-driven flows. J. Comp. Phys., 307:460-475, 2016.

[11] J. Droniou, R. Eymard, T. Gallouet, and R. Herbin. A unified approach to mimetic finite difference, hybrid finite volume and mixed finite volume method. Math. Model. Methods Appl. Sci., 20(2):1-31, 2010.

[12] J. Droniou and C. Le Potier. Construction and convergence study of schemes preserving the elliptic local maximum principle. SIAM J. Numer. Anal., 49(2):459-490, 2011.

[13] M.G. Edwards. Unstructured, control-volume distributed, full-tensor finite volume schemes with flow based grids. Comput. Geosci., 6:433-452, 2002.

[14] H.A. Friis and M.G. Edwards. A family of MPFA finite-volume schemes with full pressure support for the general tensor pressure equation on cell-centered triangular grids. J. Comput. Phys., 230:205-231, 2011.

[15] J. Karátson, S. Korotov, and M. Krizek. On discrete maximum principles for nonlinear elliptic problems. Mathematics and Computers in Simulation, 76(1-3):99-108, 2007.

[16] E.T. Karra, S.L. Painter, and P.C. Lichtner. Three-phase numerical model for subsurface hydrology in permafrostaffected regions (pflotran-ice v1.0). The Cryosphere, 8(5):1935-1950, 2014.

[17] R.A. Klause and R. Winther. Convergence of multipoint flux approximations on quadrilateral grids. Numer. Meth. Partial Diff. Eqns., 22:1438-1454, 2006.

[18] C. Le Potier. Schema volumes finis monotone pour des operateurs de diffusion fortement anisotropes sur des maillages de triangle non structures. Comptes Rendus Mathematique, 341:787-792, 2005.

[19] S.H. Lee, Y. Efendiev, and H.A. Tchelepi. Hybrid upwind discretization of nonlinear two-phase flow with gravity. Advances in Water Resources, 82:27-38, 2015.

[20] K. Lipnikov, G. Manzini, and M. Shashkov. Mimetic finite difference method. J. Comput. Phys., 257(PartB):1163-1228, 2014.

[21] K. Lipnikov, D. Moulton, and D. Svyatskiy. New preconditioning strategy for jacobian-free solvers for variably saturated flows with richards' equation. Advances in Water Resources, 2016. in press.

[22] K. Lipnikov, D. Svyatskiy, and Y. Vassilevski. Interpolation-free monotone finite volume method for diffusion equations on polygonal meshes. J. Comput. Phys., 228(3):703-716, 2009.

[23] K. Lipnikov, D. Svyatskiy, and Y. Vassilevski. A monotone finite volume method for advection-diffusion equations on unstructured polygonal meshes. J. Comput. Phys., 229(11):4017-4032, 2010.

[24] K. Lipnikov, D. Svyatskiy, and Y. Vassilevski. Minimal stencil finite volume scheme with the discrete maximum principle. Russ. J. Numer. Anal. Math. Modelling, 27(4):369-385, 2012.

[25] P.A. Lott, H.F. Walker, C.S. Woodward, and U.M. Yang. An accelerated picard method for nonlinear systems related to variably saturated flow. Advances in Water Resources, 38:92-101, 2012. 
[26] G. Manzini and e. Bertolazzi. A second-order maximum principle preserving finite volume method for steady convection-diffusion problems. SIAM J. Numer. Anal., 43(5):2172-2199, 2005.

[27] O. Misiats and K. Lipnikov. Second-order accurate monotone finite volume scheme for Richards' equation. $J$. Comp. Phys., 239:123-137, 2013.

[28] K Nikitin, K Terekhov, and Y Vassilevski. A monotone nonlinear finite volume method for diffusion equations and multiphase flows. Comput. Geosciences, 18(3-4):311-324, 2014.

[29] K. Nikitin and Y. Vassilevski. A monotone nonlinear finite volume method for advection-diffusion equations on unstructured polyhedral meshes in 3D. Russian J. Numer. Anal. Math. Modelling, 25(4):335-358, 2010.

[30] J. M. Nordbotten, I. Aavatsmark, and G. T. Eigestad. Monotonicity of control volume methods. Numer. Math., 106(2):255-288, 2007.

[31] L.A. Richards. Capillary conduction of liquids through porous mediums. Physics 1, 5:318-333, 1931.

[32] M. G. Schaap and F. J. Leij. Database-related accuracy and uncertainty of pedotransfer functions. Soil Science, 163(10):765-779, 1998.

[33] M Schneider, B Flemisch, and R Helmig. Monotone nonlinear finite-volume method for nonisothermal twophase two-component flow in porous media. Int. J. Numer. Meth. Fluids, 00:1-36, 2016.

[34] Z. Sheng and G. Yuan. The finite volume scheme preserving extremum principle for diffusion equations on polygonal meshes. J. Comput. Phys., 230(7):2588-2604, 2011.

[35] Painter S.L., Coon E.T., Atchley A., Berndt M., Garimella R., Moulton D., Svyatskiy D., and Wilson C. J. Integrated surface/subsurface permafrost thermal hydrology: Model formulation and proof-of-concept simulations. Water Resour. Res., 52, 2016.

[36] G. Stoyan. On maximum principles for monotone matrices. Linear Algebra Appl., 78:147-161, 1986.

[37] M. van Genuchten. A closed-form equation for predicting the hydraulic conductivity of unsaturated soils. Soil Sci. Soc. Am. J., 44:892-898, 1980.

[38] H. F. Walker and P. Ni. Anderson acceleration for fixed-point iterations. SIAM Journal on Numerical Analysis, 49(4):1715-1735, 2011.

[39] M. Wheeler and Yotov I. A multipoint flux mixed finite element. SINUM, 44:2082-2106, 2006.

[40] G. Yuan and Z. Sheng. Monotone finite volume schemes for diffusion equations on polygonal meshes. J. Comput. Phys., 227:6288-6312, 2008. 